\title{
A DETERMINATION OF \\ THE THICK DISK CHEMICAL ABUNDANCE DISTRIBUTION: IMPLICATIONS FOR GALAXY EVOLUTION
}

\author{
GERARD GILMORE \\ Institute of Astronomy \\ Madingley Road, Cambridge CB3 0HA \\ England, UK \\ ROSEMARY F.G. WYSE \\ Center for Particle Astrophysics \\ 301 Le Conte Hall \\ University of California \\ Berkeley, CA 94720 \\ USA \\ and \\ Department of Physics and Astronomy ${ }^{1}$ \\ The Johns Hopkins University \\ Baltimore, MD 21218 \\ USA \\ J. BRYN JONES \\ Department of Physics and Astronomy \\ University of Wales, College of Cardiff \\ Cardiff, CF4 3TH \\ Wales, UK
}

1 Permanent Address 


\begin{abstract}
We present a determination of the thick disk iron abundance distribution obtained from an in situ sample of $\mathrm{F} / \mathrm{G}$ stars. These stars are faint, $15 \lesssim V \lesssim 18$, selected on the basis of color, being a subset of the larger survey of Gilmore and Wyse designed to determine the properties of the stellar populations several kiloparsecs from the Sun. The fields studied in the present paper probe the iron abundance distribution of the stellar populations of the Galaxy at 500-3000pc above the plane, at the solar Galactocentric distance. The derived chemical abundance distributions are consistent with no metallicity gradients in the thick disk over this range of vertical distance, and with an iron abundance distribution for the thick disk that has a peak at -0.7 dex. The lack of a vertical gradient argues against slow, dissipational settling as a mechanism for the formation of the thick disk. The photometric and metallicity data support a turn-off of the thick disk that is comparable in age to the metal-rich globular clusters, or $\gtrsim 12 \mathrm{Gyr}$ and are consistent with a spread to older ages.
\end{abstract}




\section{INTRODUCTION}

The metallicity distribution of complete samples of long-lived stars has long been recognised as providing unique constraints on the early stages of chemical evolution of the Galaxy. The main sequence lifetime of $\mathrm{F} / \mathrm{G}$ dwarf stars is greater than the age of the Galaxy and hence the chemical-abundance distribution function of such stars provides an integrated record of the chemical-enrichment history without the need for modeldependent corrections for dead stars ( $c f$. van den Bergh 1962; Schmidt 1963; Tinsley 1980). Pioneering studies focussed on the only reasonably-complete sample available, which is that for stars in the immediate solar neighborhood; in effect stars within about $30 \mathrm{pc}$ of the Sun. These samples have been sufficiently small that reliable study of any stellar population whose kinematics are such that member stars spend only a small fraction of an orbit in the solar neighborhood has necessarily been difficult. This is potentially a serious restriction, as such stars might in principle be a major contributor to the stellar population in a valid, representative volume of the Galaxy. In addition, intrinsically-rare stellar populations may be missed entirely.

The present paper extends previous work by analysing an in situ sample of $\mathrm{F} / \mathrm{G}$ dwarfs with spectroscopically-determined iron abundances, at distances up to $5 \mathrm{kpc}$ from the Sun. A companion paper presents a determination of the solar neighborhood metallicity distribution derived from new, high-precision intermediate-band photometry. The combination of these data sets provides a composite distribution function which is the most reliable presently-available description of the integrated distribution of chemical abundances in a column through the local Milky Way.

\section{CHOICE OF STELLAR TRACERS}

The ideal tracer of Galactic Structure is one which is selected without any biases, does not suffer fom stellar age-dependent selection effects, is representative of the underlying populations, and is easily observable. Historically, the need for easy observation restricted studies to the immediate solar neighborhood. The primary limitation of the nearby star sample is its small size. This inevitably means that stars which are either intrinsically rare - such as halo population subdwarfs - and stars which are common but whose spatial distribution is such that their local volume density is small - such as thick disk stars - are poorly represented. Most recent and current efforts to extend present local volume-limited samples to include minority populations have, for practical observational reasons, utilized kinematically-selected samples defined in the solar neighborhood, following the pioneering work of Eggen, Lynden-Bell and Sandage (1962). Subsequent correction for the kinematic biases inherent in these samples requires careful modelling ( $c f$. Norris and Ryan 1991; Ryan and Norris 1993; Aguilar et al. 1994). An in situ sample, truly representative of the dominant stellar population far from the Sun, circumvents these large, model-dependent corrections.

Several surveys of tracer stars which can be observed in situ are available. Intrinsically luminous tracers are a priori favored in terms of telescope time, but the likely candidates have other characteristics that diminish their suitability: RR Lyrae stars have intrinsic age and metallicity biases in that only stars of a given range in metallicity 
and age exist in this evolutionary stage; the accessible globular clusters are few in number; bluer horizontal branch stars are also rare, and their color distribution depends on chemical abundance and on the unidentified 'second parameter(s)'. K-giants are the most representative evolved tracers of the spheroid, and have been used extensively. However, one must first identify giant stars from among the substantially larger number of foreground $\mathrm{K}$ dwarfs with similar apparent magnitudes and colors.

A desirable solution to these limitations, which has become feasible with current multi-object spectroscopic systems and large-scale photometric surveys, is to identify and study F/G dwarfs to significant distances from the Sun. This is the solution which we have adopted. Chemical abundances for these stars provide the integrated record of the star formation and enrichment history during the early stages of Galaxy formation, analogous to the local G-dwarf distribution.

The photometric catalogs from which the present samples are selected are derived from photographic plates from the UK Schmidt Telescope, and from the Las Campanas Observatory $2.5 \mathrm{~m}$ telescope. These plates were scanned with the COSMOS and APM measuring machines, and calibrated using what have since become standard procedures. We have defined area-complete samples in several fields chosen specifically to optimise a Galactic structure analysis; those discussed here are the South Galactic Pole and UK Schmidt Field 117, at $(\ell, b)=(270,-45)$. These two lines-of-sight are such that for the photometric definition as given below, we select stars at the same Galactocentric distance projected onto the plane, but at several kiloparsecs from the plane. The fields were also selected to have low reddening from the Burstein and Heiles (1982) HI maps.

The present samples were selected to have colors in the ranges $0.2 \lesssim \mathrm{B}-\mathrm{V} \lesssim 1.0$, and $15 \lesssim \mathrm{V} \lesssim 18$. The color range is designed to be wide enough to ensure that neither young metal-poor stars, which may be bluer than the dominant old metal-poor turnoff near $\mathrm{B}-\mathrm{V}=0.4$, nor very old metal-rich stars, whose turnoff color may be much redder than the turnoff of a metal-rich globular cluster, near $\mathrm{B}-\mathrm{V}=0.5$, are excluded. Standard star-count models indicate that the color-magnitude range isolated in the present survey should contain predominately thick-disk stars, with these making up at least $50 \%$ of the total sample. Thin-disk stars should make up some $20 \%$ of the total, with these being found almost exclusively at the reddest colors, while halo stars make up the remainder. (This prediction of course is the basis for our photometric and kinematic survey with this selection function.) These numbers are not accurately known until an adequate sample of kinematic and chemical abundance data is available. Indications to date however, based on chemical abundance, kinematic and astrometric data, are that these models over-predict the actual number of halo stars by a factor of order five (e.g. Cayrel et al. 1991a,b; Friel 1987; Perrin et al. 1994). With that correction, one expects the thick disk to make up perhaps $70 \%$ of the present sample, with the remainder being predominately old disk stars near the red edge of the sample selection.

Calibration of photographic wide-field photometry is a complex subject; the methodology is described by Gilmore (1984), while specific examples of the techniques in practice, including application to the South Galactic Pole field discussed here, are 
described in detail in Reid and Gilmore (1982), Gilmore and Reid (1983), Gilmore, Reid and Hewett (1985), Gilmore and Wyse (1985) and Kuijken and Gilmore (1989). The photometric standards upon which the calibration is based are described in those references, and in Stobie, Gilmore and Reid (1985) and Stobie, Sagar and Gilmore (1985). A detailed presentation of the photometry in this survey, including that in several other fields in which abundance data are not yet available, will be presented elsewhere. For present purposes it is sufficient to note that the external calibration, i.e. a comparison with standard stars to define the Pogson magnitude scale, has been checked from independent CCD data by Gilmore and Wyse (1985). They showed that the zero point and magnitude scale of at least the SGP dataset were well established, with error in scale and zero point being less than a few percent. That is, the data are accurate. We now consider precision.

Determination of the internal photometric error, i.e. the scatter in photometry, is extremely difficult to quantify reliably. This has been discussed for some of the data of relevance here by Gilmore, Reid and Hewett (1985) and by Reid and Gilmore (1982). A more detailed discussion, based on comparison of the independent Las Campanas and UK Schmidt data for the same fields, will be presented with the full photometric survey results. These studies are in agreement that the South Galactic Pole data are characterised by a random photometric error of $0 .{ }^{m} 05$ in $\mathrm{B}-\mathrm{V}$ color, and $0 .{ }^{m} 07$ in $\mathrm{V}-\mathrm{I}$ color, for $\mathrm{V}$ brighter than roughly $\mathrm{V}=17.5$. At fainter magnitudes a noisy tail to the error distribution is evident. Since the precise location of the magnitude limit beneath which the photometry is well defined by a single Gaussian measuring error, without an over-populated tail to the error distribution, is very important for present (chemical abundances) purposes; we consider this in more detail in the next section. In the second field, F117, the data indicate that the error distributions in both magnitude and color are well described by a single Gaussian with dispersion in color of $0 .{ }^{m} 05$ in $\mathrm{B}-\mathrm{V}$ and $0 .^{m} 07$ in V-I, to at least V=18 (Gilmore, Reid and Hewett 1985), or beyond the limit of the data discussed in this paper.

The major telescope used for the spectroscopy was the $4 \mathrm{~m}$ Anglo-Australian Telescope, first with the fibre plugboard system (FOCAP), then with the automated positioning fibre system (AUTOFIB), with the IPCS as detector. Several thousand independent spectra of program stars, distributed in the various fields have been obtained. These spectra are typically of $\sim 1.5 \AA$ resolution, covering the wavelength range $4000-5000 \AA$, with a signal to noise ratio of around 10 for program stars. The primary motivation for these spectra is to provide radial velocities accurate to about $10 \mathrm{kms}^{-1}$; the kinematics derived from the radial velocity distributions are discussed in separate papers (Gilmore and Wyse, in preparation) as is the analysis of the photometry in all the fields. Although the spectra were not intended to be useful for abundance derivations, it proved possible to derive reliable abundances from a subset of the main sample, namely the relatively high signal-to-noise spectra of the cooler stars, using a new method described in detail elsewhere (Jones, Gilmore and Wyse 1995, hereafter JGW) and discussed briefly below. Here we focus on the chemical abundance distributions derived from those data. 
The selection of stars for spectroscopic observation is designed to provide clearlydefined samples limited by area and magnitude. A more complex selection function has arisen in the subsample for which we are able to derive chemical abundances. The most important additional parameter in determination of abundances is signal-noise ratio of the spectrum, with a color-dependence of the useful limiting value, as detailed in the next section. The effect of this limit is that observations during periods of unusually good and unusually bad seeing and clouds are favoured. Good seeing clearly captures more photons, while in very bad seeing and clouds brighter stars and long exposures were selected, to obtain some data. The effect of poor conditions is clearly seen in the data for F117, where a few stars much brighter than the majority have been observed successfully. In all cases, the selection of which stars to observe was made from the available area- and color-complete catalogs without systematic color bias. The requirement that signal-noise be higher for hotter stars does, however, lead to a systematic bias, which we discuss and correct in detail below.

\section{THE IRON ABUNDANCE DISTRIBUTION OF F/G DWARFS AT SEVERAL KILOPARSECS FROM THE PLANE}

\subsection{The Iron Abundance Indicator}

The spectra of the F/G stars are of moderate resolution and signal-to-noise, being optimised for efficient measurement of radial velocities. The combination of resolution and signal-to-noise ratio is lower than is typically used for abundance determinations. In addition, the spectra were obtained through small circular apertures (the fibers) at a wide variety of zenith distances, and with variable centering precison. Thus the continuum flux distribution in the spectra is unreliable over wavelength ranges greater than a few tens of angstroms. This combination provides a challenge for the determination of chemical abundances. Jones (1991; JGW) developed an analysis technique appropriate to the derivation of true iron abundances from these spectra. Full details are given in these references, with only a brief summary here.

The method utilizes narrow band 'photometric' indices, analogous to equivalent width measurements, formed by integrating the flux in a narrow spectral region relative to adjacent continuum regions. The important features of the method are that the spectral regions are chosen to match the resolution of the data, to contain only Fe I lines which lie on the linear part of the curve of growth, and which have nearby regions of pseudo-continuum. The indices were identified and calibrated from synthetic spectra, utilising a grid of 100 synthetic spectra which were derived from a scaled solar model atmosphere.

Four abundance indices were defined which measure absorption from very strong Fe I lines; the pressure-sensitivity of the wings of these lines means that the indices are also dependent on surface gravity to an appreciable extent. Seven abundance indices were defined which measure absorption caused by weaker Fe I lines; in the stars of interest to this work, these exhibit little gravity-sensitivity. Five indices have been identified which measure the relative strengths of absorption lines of ionised and neutral species. These are strongly sensitive to gravity. From these 16 indices a compound iron 
abundance indicator was defined using all metallicity-sensitive indices, and the iron abundances derived using this compound indicator.

Given a photometrically-determined effective temperature, then if the spectrum is not too noisy, $\mathrm{S} / \mathrm{N} \gtrsim 25$ in $1 \AA$ pixels, it is possible with this technique to solve for both iron abundance and surface gravity. This is indeed possible for the standard stars observed, checking the calibration of the system, but for most of the program stars we are forced to adopt a priori a value for the surface gravity; in this case, we assume the star is near the main sequence, and adopt $\log g=4.0$. A value of $\xi=1.5 \mathrm{~km} / \mathrm{s}$ for microturbulence was adopted for all stars.

Stellar effective temperatures are derived from photographic V-I photometry. A new determination of the $(\mathrm{V}-\mathrm{I})$ - $\mathrm{T}_{\text {eff }}$ relation was made from published data, as explained in JGW. No appreciable metallicity dependence was found, with the adopted calibration being

$$
\frac{5040}{\mathrm{~T}_{\text {eff }}}=0.484( \pm 0.010)+0.581( \pm 0.014)(\mathrm{V}-\mathrm{I})
$$

Thus for the F/G stars studied here, the typical uncertainty in effective temperature is $\sim 200 \mathrm{~K}$. The uncertainty in iron abundance resulting from given uncertainty in effective temperature was derived from detailed Monte Carlo simulations, and is given for reference in Table 1. The uncertainties in log metallicity scale approximately linearly with temperature uncertainty. Note that in general the estimate of the metallicity is more sensitive to temperature for lower metallicity stars, for fixed uncertainty in effective temperature, and at fixed signal-to-noise, for hotter stars. A typical star in our sample has $T_{e f f}=5500 \mathrm{~K}$ and for this temperature an error of $100 \mathrm{~K}$ yields an uncertainty in $[\mathrm{Fe} / \mathrm{H}]$ of 0.09 dex; an error of 1.0 in $\log g$ yields an uncertainty of 0.15 dex in $[\mathrm{Fe} / \mathrm{H}]$, and an error of $0.5 \mathrm{~km} / \mathrm{s}$ in the microturbulence parameter yields an uncertainty of 0.12 dex at $[\mathrm{Fe} / \mathrm{H}]=0.0$, and of 0.03 dex at $[\mathrm{Fe} / \mathrm{H}]=-1.5$. Thus the typical uncertainty in the derived $[\mathrm{Fe} / \mathrm{H}]$ may be expected to be $\sim 0.2 \mathrm{dex}$.

The zero-point of the iron abundance calibration was tested first by application to many very high $\mathrm{S} / \mathrm{N}$ ratio spectra of the twilight sky (scattered solar light) spectrum; the compound indicator provided a median value of $[\mathrm{Fe} / \mathrm{H}]=-0.14$, which was adopted as a zero-point correction. Comparison of the iron abundances we derived from our observations of standard stars with published $[\mathrm{Fe} / \mathrm{H}]$ data (mostly from Laird, Carney and Latham 1988; hereafter LCL) provided a mean difference, defined as the new derived value of this paper minus the LCL published value, of -0.04 dex, sigma $=0.13$ dex, for 16 stars in the range $-1.2<[\mathrm{Fe} / \mathrm{H}]<0$ (one extreme outlier removed). The rms difference increased to $0.24 \mathrm{dex}$, with a mean offset of $+0.06 \mathrm{dex}$, if more metal-poor stars were included. Thus the uncertainty in derived $[\mathrm{Fe} / \mathrm{H}]$ expected from the Monte Carlo simulations is consistent with a direct comparison with observations.

\subsection{Distances}


Distances to the stars can be derived by photometric parallax, assuming the stars are in a known evolutionary stage and single (binarity is discussed below), given a calibration of luminosity on iron abundance. We will assume the stars to be unevolved (but see below), and adopt the metallicity-luminosity calibration of LCL, which is given in terms of the offset in absolute $\mathrm{V}$ magnitude from the Hyades main sequence, for which they obtain

$$
\mathrm{M}_{\mathrm{V}}(\text { Hyades })=5.64(\mathrm{~B}-\mathrm{V})+0.11 .
$$

The metallicity-dependent offset from this fiducial sequence that LCL derive $\left(\Delta \mathrm{M}_{\mathrm{V}}^{\mathrm{H}}\right)$ is, for a star of given $(\mathrm{B}-\mathrm{V})$ color and given UV excess $\delta$ :

$$
\Delta \mathrm{M}_{\mathrm{V}}^{\mathrm{H}}=\left[\frac{2.31-1.04(\mathrm{~B}-\mathrm{V})}{1.594}\right]\left[-0.6888 \delta+53.14 \delta^{2}-97.004 \delta^{3}\right] .
$$

LCL state this calibration to be valid for $\delta \leq 0.25$, which equals $[\mathrm{Fe} / \mathrm{H}]=-1.75$ dex with the Carney (1979) transformation of $\delta$ into $[\mathrm{Fe} / \mathrm{H}]$, as used by LCL:

$$
[\mathrm{Fe} / \mathrm{H}]=0.11-2.90 \delta-18.68 \delta^{2} .
$$

$[\mathrm{Fe} / \mathrm{H}]=-1.75$ is the mean metallicity of the LCL calibrating subdwarfs; note that their calibrators for this relationship extend down to $[\mathrm{Fe} / \mathrm{H}]=-2.45$ dex. The majority of our program stars have derived metallicities which are well within their suggested range for this relationship to be valid, $>-1.75$ dex, and certainly within that of the calibrating subdwarfs (see below). LCL provide an alternative expression for stars more metalpoor than $-1.75 \mathrm{dex}$, which they obtained from model isochrones. This latter technique provides distances for our metal-poor stars which are in general within 10\% (larger) of those derived from the former, observationally anchored technique. We have chosen to apply the former technique to our entire sample. The typical error in our derived $[\mathrm{Fe} / \mathrm{H}]$ of $\sim 0.2 \mathrm{dex}$ leads to a random uncertainty of $\sim 20 \%$ in distance estimate. There are also sources of systematic error.

The possibility that the 'stars' are instead unresolved binaries means that the distances derived as above are underestimates, i.e. the uncertainty in distance has a systematic, as well as a random, component. A typical binary system in the present sample may be taken to have a metallicity $[\mathrm{Fe} / \mathrm{H}] \sim-0.5$ dex (see below) which with our distance estimator leads to a photometrically-derived absolute magnitude of the binary, $\mathrm{M}_{V, P}$, given in terms of the composite color of the binary as:

$$
\mathrm{M}_{V, P}=5.31(\mathrm{~B}-\mathrm{V})_{\text {composite }}+1.845 .
$$

Following Kroupa, Tout and Gilmore (1991) we can use this to express the factor by which the photometrically-derived distance, $d_{P}$, underestimates the true distance, $d$, in terms of the absolute $\mathrm{V}$ magnitudes of the putative binary components, $\mathrm{M}_{V 1}$ and $\mathrm{M}_{V 2}$ respectively, as :

$$
\frac{d_{P}}{d}=\frac{\left(10^{-0.475 \mathrm{M}_{V 1}}+10^{-0.475 \mathrm{M}_{V 2}}\right)^{2.655}}{\left(10^{-0.4 \mathrm{M}_{V 1}}+10^{-0.4 \mathrm{M}_{V 2}}\right)^{3.155}} .
$$


Equal-mass binaries provide the worst case, with the estimated distance being a factor of $1.414(=\sqrt{ } 2)$ smaller than the true distance. The results of Kroupa, Tout and Gilmore $(1991 ; 1993)$ favor a high binary fraction, $\gtrsim 50 \%$, but with components chosen independently from the mass function, so that equal masses rarely occur. A mass ratio very different from unity of course means that the systematic errors in the distance estimator are greatly reduced from the worst case $40 \%$, and the typical errors are more likely to be much smaller. Indeed, for stars of the $\mathrm{V}-\mathrm{I}$ color of the present sample, Monte-Carlo simulations suggest a systematic mean error of only $\sim 5 \%$ in distance (Kroupa, Tout and Gilmore 1993, their Figure 8).

A further assumption that we have made which, if inappropriate, would systematically underestimate the true distance, is that the stars are all on the main sequence. The predictions of our star count models in the lines-of-sight of our samples are that typically $\lesssim 25 \%$ of stars in the color and magnitude ranges here are evolved, with most of these stars being subgiants rather than red giants or red horizontal-branch stars. The error in distance estimate which results from assigning these stars to the main sequence obviously depends on color. However, the phase of subgiant evolution that has by far the longest duration is the initial vertical evolution (Iben 1967), when the star is closest to the zero-age main sequence. The error for a star in this evolutionary stage is about a $20 \%$ underestimate in distance.

This systematic error applies of course only to those stars with colors equal to those of the main-sequence turnoff, which is near $\mathrm{B}-\mathrm{V}=0.4$ for metal-poor stars, and $\mathrm{B}-\mathrm{V}=0.55$ for typical thick disk and very old disk stars with the abundances we derive below. In our South Galactic Pole data set, which is that with the largest sample, some $30 \%$ of stars have $\mathrm{B}-\mathrm{V} \leq 0.60$. Fortunately, as discussed below, there is no significant correlation of abundance and color for stars redder than $\mathrm{B}-\mathrm{V}=0.5$, so this distance uncertainty will not affect our conclusions below.

Since we do not know a priori which of these stars is a binary, or an evolved star, we should assign the above systematic errors to the entire sample. Thus we expect that the distances derived here have about $20 \%$ random errors, and may be underestimated, by perhaps $20-30 \%$, especially for the bluer stars in the sample.

\subsection{The South Galactic Pole Sample}

The method outlined above was applied to derive $[\mathrm{Fe} / \mathrm{H}]$ abundances for a sample of 133 stars in the South Galactic Pole (SGP) field. It should be noted that these are true iron abundances. The observed and derived data for these stars are given in Table 2. There were 3 stars which were observed with enough $\mathrm{S} / \mathrm{N}$ on two occasions to allow separate metallicity estimates, providing a (weak) internal check on the technique. These stars show a mean offset of $0.13 \mathrm{dex}$, and a dispersion of $0.2 \mathrm{dex}$, in agreement with the expected uncertainties. The value of $[\mathrm{Fe} / \mathrm{H}]$ quoted in the table for these three stars is that obtained from the co-added data.

The basic photometric data for the program stars are shown in Figure 1(a) and (b), as $\mathrm{V}, \mathrm{B}-\mathrm{V}$ and $\mathrm{V}, \mathrm{V}-\mathrm{I}$ color-magnitude diagrams. The $\mathrm{B}-\mathrm{V}, \mathrm{V}-\mathrm{I}$ two color diagram for these stars is shown in Figure 2, together with the mean relation found by 
Reid and Gilmore (1982) from main sequence E-region standard stars (apparently bright and hence nearby and expected to have close to solar metallicity). The large scatter of the data points in this last plot is due to a combination of photometric errors and true metallicity spread. $\mathrm{B}-\mathrm{V}$ is metallicity-dependent, being bluer for lower metallicity (for constant stellar $\mathrm{T}_{e f f}$ ), while $\mathrm{V}-\mathrm{I}$ is primarily a measurement of temperature. The metallicity dependence of the two-color diagram for our sample may be quantified by using the stellar evolution models of VandenBerg and Bell (1985; Y=0.20), and of VandenBerg $(1985 ; \mathrm{Y}=0.25)$. Figure 3 shows the colors of a one solar mass star of fixed effective temperature, $\mathrm{T}=5786 \mathrm{~K}$ (chosen to minimise the interpolation required), but a range of metallicities (and hence age, but always many Gyr). As can be seen, the models get bluer by almost $0 .^{m} 1$ in $\mathrm{B}-\mathrm{V}$, with negligible change in $\mathrm{V}-\mathrm{I}$, as the metallicity is decreased from solar to one-tenth solar.

The importance of photometric errors may be seen more clearly with a larger sample; Figure 4 shows the V, B-V data for all stars in our magnitude range in several $40^{\prime}$ fibre fields. The very blue stars, $\mathrm{B}-\mathrm{V} \lesssim 0.3$, occur predominantly for $\mathrm{V} \gtrsim 17.3$. As discussed in section 2 above, these faint magnitudes are where the photometric errors are becoming too large for present purposes (see Table 1). The metallicity analysis is thus restricted to only stars with $\mathrm{V}<17.30$, which incidentally also has the effect of removing all stars bluer than $\mathrm{B}-\mathrm{V}=0.4$, and in total removes 32 stars from further discussion here. That the errors for the fainter stars are larger may also be seen by a plot of the $\mathrm{B}-\mathrm{V}, \mathrm{V}-\mathrm{I}$ two color diagram for all 133 stars, binned by their derived metallicities, as shown in Figure 5(a). The corresponding plot for only those stars (101 in total) brighter than $\mathrm{V}=17.30$ is shown in Figure 5(b); this latter plot shows reduced scatter, and a trend of the offset from the E-region standard star line with metallicity.

An investigation of the integrated chemical evolution of the disk requires a sample of stars of long enough main-sequence lifetimes to still be around today even if formed at early times, say 12 Gyr ago ( $c f$. the ages derived by Edvardsson et al. 1993). The $[\mathrm{Fe} / \mathrm{H}]$ data for the 101 brighter stars are shown as a scatter plot against $\mathrm{B}-\mathrm{V}$ color in Figure 6, together with the 12 Gyr turn-off positions from VandenBerg and Bell (1985; crosses) and VandenBerg (1985; asterisks). These isochrones provide an age of 14Gyr for the globular cluster 47Tuc, and it is clear from the Figure that the turnoff of the bulk of our sample is somewhat redder than the $12 \mathrm{Gyr}$ isochrone, consistent with the inference (Wyse and Gilmore 1988; Gilmore, Wyse and Kuijken 1989; Edvardsson et al. 1993) of an age of the thick disk comparable to that of the metal-rich globular clusters. ${ }^{2}$ There

2 Note the lack of metal-rich stars blueward of the theoretical turnoff positions. This does not imply a lack of younger metal-rich stars in the thin disk, but rather results from the magnitude-color selection. The stars with main-sequence lifetimes less than 12 Gyr are simply so luminous that to be seen with the apparent magnitude of the sample they would be beyond the effective edge of the thin disk (but not of the thick disk). Since essentially all thin-disk stars older than 3 Gyr have the same vertical velocity dispersion and scaleheight this entire range of ages will be represented in the tail of the thin disk that is in the sample, at redder colors. 
remains one star that lies (just) blueward of the $12 \mathrm{Gyr}$ turnoff positions; since we are interested in isolating a sample of stars that have main-sequence lifetimes greater than the present age of the disk, we have removed this star from the metallicity distribution discussed below. We will also restrict the sample to stars with $\mathrm{B}-\mathrm{V}<0.9$, further to isolate $\mathrm{F} / \mathrm{G}$ dwarfs, which removes a further two stars. This leaves a sample of $98 \mathrm{~F} / \mathrm{G}$ dwarfs in our SGP sample, with $0.4<\mathrm{B}-\mathrm{V}<0.9$.

Even just considering the stars brighter than $\mathrm{V}=17.30$, comparison of Figures 1 and 4 shows that there is a clear deficiency of stars bluer than $\mathrm{B}-\mathrm{V}=0.5$ in the sample for which iron abundances were derived, compared to the available stars on the sky. This arises since it is more difficult to derive reliable abundance estimates for these hotter stars from the available low $\mathrm{S} / \mathrm{N}$ data. This bias is important since it is clear from the data that the metal-poor stars tend to be bluer, and thus the effective temperature bias will translate into a metallicity bias. This may be corrected for by renormalising the color distribution of stars in the metallicity sample to agree with that available on the sky. The number in the metallicity sample relative to the area-complete sample, in color bins of $0.1 \mathrm{mag}$ starting at $\mathrm{B}-\mathrm{V}<0.5$ and ending at $\mathrm{B}-\mathrm{V}=0.9$ is $0.35 ; 0.68 ; 0.58 ; 0.60$ and 0.73 respectively. This means that, allowing for the limitations of small number statistics, these ratios are $0.5: 1: 1: 1: 1$ and the metallicity distribution needs to be corrected only for those stars in the bluest $\mathrm{B}-\mathrm{V}$ bin, and that the stars in that bin should be given double weight in the iron abundance distribution. The resulting distribution is then free of color bias. The metallicity of the initial, biased sample is shown as the dashed histogram in Figure 7, together with the color-corrected distribution (solid histogram). The correction is clearly a small effect.

The iron-abundance distribution also needs to be corrected to derive a volumecomplete sample from our area-complete photometrically-defined sample. Distances to the stars were derived as described above. The scatter plot of $[\mathrm{Fe} / \mathrm{H}]$ against distance for the 98 stars with $\mathrm{V}<17.30$ and $0.4<\mathrm{B}-\mathrm{V}<0.9$ is shown in Figure 8. The scatter plot of $\mathrm{B}-\mathrm{V}$ versus distance for these stars is shown in Figure 9, together with the limits set by our pruned magnitude range, for given metallicity, with the solid lines corresponding to the faint limit of the sample and the dashed lines to the bright limit. The sample is complete for a given iron abundance within the volume defined by the two distance limits. It can be seen from combination of Figures 8 and 9 that the more metal-rich stars observed tend to be found preferentially near the inner distance limit of the sample selection criteria. This is despite the fact that were metal-rich stars to exist at large distances, they would be favored by the selection in magnitude and color. Thus one may infer immediately that the more metal-poor stars are in a more spatially-extended distribution than the more metal-rich stars.

The metallicity distribution of a volume-complete sample may be derived from the observed distribution (after the correction for color bias in the metallicity determinations described above) by calculating the volume accessible to a given metallicity bin at its characteristic distance, and then bringing all metallicity bins to a common, fiducial distance from the plane. Note that we prefer the method of direct integration to that of $\mathrm{V} / \mathrm{V}_{\max }^{\prime}$; the $\mathrm{V} / \mathrm{V}_{\max }^{\prime}$ method is less appropriate here as there is a mix of stellar 
populations with overlapping abundance distributions but different spatial distributions. In our simplest case below all stars are assumed to be thick-disk stars, and our direct integration method and the $\mathrm{V} / \mathrm{V}_{\max }^{\prime}$ method are equivalent.

The volume accessible to each metallicity bin is the integral of the volume element between the maximum and minimum distances shown in Figure 9, achieved by first integrating at a fixed color, and then simply summing over color, since corrections for color bias have already been applied. The characteristic distance for each metallicity bin is obtained by integrating the line element, weighted by the density profile of the stars, between the limits set by the color selection; this is necessary since the stars do not uniformly occupy the accessible volume. Thus some model-dependency enters through choice of density profile; use of the individual distances derived above to determine a mean distance is hampered by the small numbers in the metal-poor and metal-rich ranges of the sample. The present small sample is obviously not well-suited for the determination of self-consistent density profiles. We adopted the thin disk and thick disk scaleheights obtained by Kuijken and Gilmore (1989) for K dwarfs at distances comparable to those probed by the present sample, 300-4000pc, viz. 250pc for the thin disk and 1000pc for the thick disk, and have modelled the stellar halo by an exponential profile with scaleheight $4000 \mathrm{pc}$, much larger than either of the disks.

As discussed in section 2 above, the present sample is expected to be dominated by thick disk stars, with some contribution from the stellar halo presumably mostly at the metal-weak end, and from the thin disk at the metal-rich end. As noted above, the distance distribution of the sample does imply a more extended spatial distribution for the more metal-poor stars. We tested the sensitivity to the density profiles by calculating the corrections in two ways - first by simply adopting the thick disk density profile for all metallicity bins, and secondly by adopting the stellar halo density profile for stars more metal-poor than -1 dex, the thick disk density profile for all stars above this until $-0.4 \mathrm{dex}$, allowing $10 \%$ thin disk contribution to the stars with $-0.4<[\mathrm{Fe} / \mathrm{H}]<0$, and assigning all stars more metal rich than solar to the thin disk. These percentages are in broad agreement with the expectations from star-count models. In general one expects the results to be rather insensitive to the choice of density profile except for the most metal-rich bins, where the thin disk density profile is applied and the depth of the sample is a significant number of scaleheights. This expectation is borne out by Table 3, which gives the resultant relative numbers of stars per unit volume at a fiducial distance of $1500 \mathrm{pc}$, chosen to be close to the middle of the range of distances sampled by the data. The histograms of the volume-complete metallicity distributions obtained these two ways are shown in Figure 10. Note that the metallicity distribution of the thin disk is of course better constrained by a sample more concentrated to the plane; such is the local Gliese catalog sample analysed in the companion paper. Indeed, both the metal-poor bins, $[\mathrm{Fe} / \mathrm{H}]<-1$, and the metal-rich bins, $[\mathrm{Fe} / \mathrm{H}]>$ solar, are based on rather a small number of stars, so even although they are apparently well-populated in the corrected distributions, their error bars are large.

\subsection{The Rotation Field}


Iron abundances were obtained for a sample of 87 stars in the direction of $(\ell, b)=$ $(270,-45)$, UK Schmidt Field 117, as tabulated in Table 4. There were 3 stars which were observed with enough $\mathrm{S} / \mathrm{N}$ on two occasions to allow a metallicity estimate to be made, providing an internal check on the calibration. This yielded a mean offset of 0.05 dex, again with a dispersion of $0.2 \mathrm{dex}$, and again the value quoted in the table is that obtained from the co-added data.

Following the analysis of the SGP stars, the $\mathrm{V}, \mathrm{B}-\mathrm{V}$ color magnitude diagram of the sample with derived iron abundances is shown in Figure 11(a), and the V, V-I data are shown in Figure 11(b). Note the few stars brighter than $\mathrm{V}=16$ which were observed as a poor-seeing back-up; these were drawn at random from the same color range as the fainter stars. The $\mathrm{V}, \mathrm{B}-\mathrm{V}$ data for representative entire fibre fields are shown in Figure 12. Again, there is a deficiency of blue stars, $\mathrm{B}-\mathrm{V} \lesssim 0.55$, in the metallicity sample. Although there is no trend of $[\mathrm{Fe} / \mathrm{H}]$ with $\mathrm{B}-\mathrm{V}$ color, for $\mathrm{B}-\mathrm{V} \gtrsim 0.6$ where we have a large enough sample in this field, comparison with the SGP data suggests that there may be an effect for bluer stars, and lower metallicities $[\mathrm{Fe} / \mathrm{H}] \lesssim-1$ dex, and we would not be aware of it here. The metallicity distribution may be unaffected by the color bias, but conservatively one should treat the derived number of stars in this field with $[\mathrm{Fe} / \mathrm{H}] \lesssim-1$ dex as lower limits only (as we discuss below, the fact that there is little difference between the distribution in the SGP and that found in this field suggests that any remaining bias against metal-poor stars is a small effect). For convenience, we note that binning the data into $0 .{ }^{m} 1$ ranges of $\mathrm{B}-\mathrm{V}$, centered on $0.55,0.65,0.75$ and 0.85 , the ratios of the number of stars in the metallicity sample to that in the entire fibre field are $0.12 ; 0.20 ; 0.20 ; 0.10$. Since there is no trend between color and metallicity in this range the lack of constancy of this ratio is not meaningful in determining the derived metallicity distribution in this field. We also for consistency remove stars with $\mathrm{B}-\mathrm{V} \geq 0.9$, which leaves a total of 83 stars in this line-of-sight.

The two color diagram for the stars in this field with metallicity determinations is shown in Figure 13. The corresponding diagrams for sub-samples selected by iron abundance is shown in Figure 14, where there is a clear trend in offset from the Eregion standard star ridge line with decreasing metallicity. The photometry in all fields is calibrated independently, as discussed in section 2 above. Unlike the in SGP, there are no indications in this field that the errors in the photometry are unacceptable for the faint stars. However, it should be noted that the most metal-poor stars are faint, and hence the derived metallicities have larger error bars. In compensation, these bins are sparsely populated and are given low weight in the discussion below.

Following the analysis for the SGP stars, Figure 15 shows the scatter plot of B-V color versus $[\mathrm{Fe} / \mathrm{H}]$, together with the $12 \mathrm{Gyr}$ turnoff positions from VandenBerg and Bell (1985) and from VandenBerg (1985). There are no stars in this field which should be excluded by virtue of their location in this plot. The color and magnitude limits for this field are then $15.0 \leq \mathrm{V} \leq 17.87,0.5 \leq \mathrm{B}-\mathrm{V}<0.9$; as discussed in section 2 the coverage of stars within these limits is not uniform. 
The distances for the F117 stars were derived as for the SGP stars. The direction of this field is such that the planar radial Galactocentric distances of the F117 stars are not significantly different from the solar circle - the maximum planar Galactocentric distance probed by the sample is $8.6 \mathrm{kpc}$, for a solar Galactocentric distance of $8 \mathrm{kpc}$. Most stars are at distances from the Sun of $1.5 \mathrm{kpc}$, corresponding to a planar Galactocentric distance of $8.1 \mathrm{kpc}$. Comparison of the iron-abundance distribution in this field with that of the SGP is therefore not sensitive to the presence or otherwise of radial Galactic abundance gradients. The vertical height distribution of the sample in this field covers the range $0.7 \lesssim z(\mathrm{kpc}) \lesssim 1.5$, with approximately equal numbers below and above $z=1.1 \mathrm{kpc}$ ( $\operatorname{since} z=d \times \sin 45^{\circ}$ the vertical distances are trivially derived from the distances, $d$, given in the Table). The metallicity distribution in this field, when combined with the SGP data above which have equal numbers of stars above and below $1650 \mathrm{pc}$, thus provides a probe of the vertical metallicity gradient of the thick disk at the solar circle.

Figure 16 shows the stars in the $[\mathrm{Fe} / \mathrm{H}]$, distance plane, and Figure 17 the $\mathrm{B}-\mathrm{V}$, distance plane, where the lines indicate the distance limits corresponding to the sample magnitude limits for given metallicity. Corrections for the variation of the volume sampled with color and metallicity were made in the same way as the analysis of the SGP sample. Table 5 gives the binned iron-abundance distribution for the 'raw' sample, together with characteristic distances and number of stars per unit volume, at a fiducial vertical distance of $1000 \mathrm{pc}$, under the two sets of assumed density profiles. Histograms of these iron-abundance distributions are shown in Figure 18. Again, the most robust determination is for the iron-abundance distribution in the range $-1.0<[\mathrm{Fe} / \mathrm{H}]<0$, with the (noisy) data below $[\mathrm{Fe} / \mathrm{H}]=-1$ being formally a lower limit to the numbers of these stars (although as discussed in section 4 below, this is not in fact a problem in practice).

\section{DISCUSSION}

The abundance distribution at a distance of $1.0-1.5 \mathrm{kpc}$ from the Galactic Plane which we have derived above contains valuable information on the star-formation and chemical-enrichment history of the thick disk. In a complementary paper (Wyse and Gilmore 1994) we combine this distribution with a new determination of the abundance distribution in the Galactic Plane to reconsider the evolution of the Galactic disk at the solar Galactocentric radius. In a future paper we combine the present data with our larger kinematic and photometric survey and other relevant published data. Prior to those analyses, we note here some of the more obvious implications of our thick disk abundance distribution.

\subsection{Is the Thick Disk Old?}

The combination of chemical abundance and photometric data allows determination of turnoff ages. The data and isochrones shown in Figure 6 for the SGP and Figure 15 for F117 are both confirmatory evidence that the thick disk is predominately 
composed of stars which are as old as the oldest disk stars, and possibly as old as the metal-rich globular clusters ( $c f$. Nissen and Schuster 1991).

Most models of thick-disk formation suggest that there should be an age spread in this component. This will reflect the star-formation history of the precursor thin disk and/or satellite for slow or rapid kinematic heating models. Star formation in a pressuresupported thick gas disk, or models in which there is a burst of star formation during a merger event can however produce a narrow age range (although merger-induced star formation may be expected to be rather more centrally-concentrated than the thick disk is observed to be, due to gravitational torque-induced gas flows towards the central regions; Mihos and Hernquist 1994). Our present data are insufficient to investigate such possibilities, as isochrone crowding implies that high-precision, narrow-band photometric data are required for adequate age resolution at old ages. The predominant old age shown in these data does however limit all formation models to have the creation of the thick disk at early times. Thus, models invoking slow kinematic heating of thin-disk stars must appeal to special heating conditions in the early Galaxy, while merger models must have only early merger(s), or produce thick disks that consist predominantly of stars from the shredded satellite, which must itself be predominantly old.

More detailed element-ratio data allow determination of star-formation histories, initial mass functions, and interstellar-medium mixing timescales and efficiencies. These topics will be considered in some detail in a later paper. However, the fact that thick-disk stars have element ratios that reflect incorporation of iron from Type I supernovae (e.g. data of Edvardsson et al. 1993) implies a formation timescale more extended than the timescale appropriate for these supernovae, probably $\sim 1 \mathrm{Gyr}$. Indeed, the Edvardsson et al. estimates of stellar ages directly suggest a range of ages for the thick disk at the solar neighborhood (defined by the mean radius of their orbit calculated in Edvardsson et al. as being between $7 \mathrm{kpc}$ and $9 \mathrm{kpc}$ ) of at least a few Gyr, ¿ 12Gyr (note that the kinematic selection effects in that sample make it difficult to isolate thick-disk stars at other radii). It may also be noted that the Edvardsson et al. age estimates do not show any significant age gap between the thick disk and thin disk stars, as would be expected if one were to believe the combination of the (young) white dwarf ages for the thin disk and the (old) stellar evolutionary ages for the globular clusters. Further, their element ratio data do not show any indication of a hiatus in star formation - if there were a hiatus, then enrichment of oxygen, and of other alpha-elements which are primarily produced in Type II supernova exposions of massive stars, would cease for the duration of the hiatus, but enrichment in iron would continue, in line with current models of Type I supernovae (binary white dwarfs) which allow for an final explosion many Gyr after the birth of the progenitors. Stars born after a hiatus in star formation are then formed from gas which has been preferentially enriched in iron, and in particular will show low oxygen-to-iron ratios (Gilmore and Wyse 1991). However, the element ratio data of Edvardsson et al. are continuous at the solar neighborhood, as can be seen from their Figure 21 (alpha-to-iron versus age) and their Figure 22 (oxygen-to-iron ratio versus iron abundance).

\subsection{Is There a Vertical Abundance Gradient in the Thick Disk?}


A comparison between the iron-abundance distribution in the SGP (at a fiducial distance of 1500pc) and in F117 (at $z=1000 \mathrm{pc}$ ) is shown in Figure 19. It is clear that there is no significant difference between the distributions in these two fields. Further, this comparison suggests that the incompleteness for $[\mathrm{Fe} / \mathrm{H}]<-1$ dex in $\mathrm{F} 117$ cannot be a large effect. The thick disk abundance distribution in the Galactic Plane is poorly determined, but in so far as it is known may be characterised as a Gaussian with mean abundance at -0.6dex (Carney, Latham and Laird, 1989), again consistent with the in situ data presented here. Thus, the available thick disk abundance determinations as a function of distance may most easily be interpreted in terms of a constant ironabundance distribution for the thick disk, independent of height above the plane, at least over the range probed by these data. This is in agreement with the conclusions from available in situ samples of thick-disk stars with UV-excess photometric metallicity estimates (Gilmore and Wyse 1985; Majewski 1992).

In principle the absence of a significant abundance gradient is weak evidence against some models of thick disk formation. The relevant models may crudely be categorised as slow dissipative formation from gas with the same spatial distribution as the resulting thick stellar disk; slow kinematic heating of stars formed in a thin disk, or disruptive or very rapid heating of a thin disk. Scenarios of thick disk formation that invoke disruptive heating of a pre-existing thin disk, by means such as satellite galaxy accretion, will in general predict lower amplitude metallicity gradients in the thick disk than do scenarios that invoke slow dissipative settling. However, if there were a vertical abundance gradient in a thin disk before rapid heating, then current models suggest that dissipationless relaxation after merging will weaken such a metallicity gradient, but not erase it (White 1980; Hernquist and Quinn 1993), so there is not a clear dichotomy between predictions of these two scenarios.

Were the thick disk a tracer of a fairly major merging event - accretion of a satellite of mass around $10 \%$ or more of the mass of the Galactic disk (Quinn, Hernquist and Fullager 1993) - then one may expect two places of origin of the stars that are now in the thick disk, some being shredded satellite, others being originally thin Galactic disk. Satellite galaxies presently observed appear to follow a well-defined luminositymetallicity relationship that is not a simple extrapolation of that of normal galaxies (Caldwell et al. 1992). It may not be coincidence that the mean metallicity of the thick disk, $\sim-0.6$ dex, is comparable to that of the Small Magellanic Cloud today, which is probably of sufficient mass to be representative of the type of satellite that could cause a thick disk to form when accreted. Satellite galaxies of the type which surround the Milky Way today typically have metallicity of $\sim-1.5$ dex and absolute magnitudes $M_{V} \sim-12$ (Armandroff et al. 1993). Such a galaxy would probably not have sufficient impact to create a thick disk by heating, but could contribute stars by direct shredding. Of course, what is really relevant is the stellar mass of the thin disk perhaps $12 \mathrm{Gyr}$ ago, and the chemical abundance of the stars in the satellite galaxy at that epoch. Merger models clearly are able to generate complex age and abundance distributions in the thick disk. A common feature is however a weak or zero vertical abundance gradient, consistent with present observations. 
If the thick disk were formed from the thin disk in a manner which preserves the initial conditions in the thin disk then one might expect to observe a vertical abundance gradient. For example, a model which invokes slow kinematic heating of a thin disk to create the thick disk will produce a thick disk which retains memory of any agemetallicity relation which may have been established in the thin disk. Given that there is little or no evidence for any age-metallicity relation in the thin disk today, it remains unclear if this property of slow heating models provides an observational signature in the abundance gradient. Such models are better tested from age data.

\subsection{The Abundance Distribution Function at $z=1.5 \mathrm{kpc}$}

With the knowledge that the F117 metallicity distribution is indeed complete at the metal-poor end, and that there is no evidence for a significant vertical abundance gradient in the data, we are now able to combine the SGP and F117 abundance distributions into our final in situ abundance distribution. We first correct the F117 distribution function to $\mathrm{z}=1.5 \mathrm{kpc}$, for consistency with the SGP data. We do this for both the 'every star is a thick-disk star' density profile model and for the three-populations model. The distributions in the two fields do not differ significantly in either shape or number of stars in the original sample, so we can combine them by summing. The resulting distribution functions are presented in Table 6, which represents our final abundance distribution function. This distribution function is combined with an updated thin-disk metallicity distribution derived from the Gliese catalog, and the composite analysed, in a separate paper. We now consider the more metal-poor stars.

The present sample has been highly optimised for study of the in situ thick disk. Consequently, these data are not ideal for a study of either the thin disk or the stellar halo. However, one can use information on these latter populations from other sources to aid in the interpretation of the iron abundance data of the present sample. In particular, the contribution of the stellar halo to the metal-poor stars can in principle be estimated by combination of its chemical abundance distribution and the predictions of star-count models, allowing statistical isolation of the metal-poor stars in the thick disk from those in the halo.

Extension of the metallicity distribution function of the thick disk to low metallicities is a natural expectation in most scenarios for thick-disk formation. For example, should the thick disk represent the first stars to form in the disk, then one expects stars of arbitrarily low metallicities in the thick disk. The thick disk and thin disk are in fact plausibly chemically-connected from the continuity of element ratios and ages in the data of Edvardsson et al. (1993). The kinematics of thick and thin disk also suggest an evolutionary connection - as seen in the similarity of their angular-momentum distributions (Wyse and Gilmore 1992) and in the locations of thick- and thin-disk stars in the the orbital eccentricity-metallicity plane (most recently in Twarog and AnthonyTwarog 1994). Thus it is plausible that the thick disk is the chemical precursor to the thin disk, and that the earliest, and most metal-poor, disk stars to form in the Galaxy are now in the thick disk. 
The number of such very metal-poor stars, or more correctly the shape and normalisation of the metal-weak tail to the thick disk (and the thin disk, for that matter) cannot be predicted reliably from first principles. Indeed, this difficulty is simply a restatement of the 'G-dwarf problem'. Depending on details of such things as pre-enrichment from previous stellar generations, gas flows, inhomogeneities etc., one may have any distribution from that of the Simple Model, which has a substantial metalweak tail, to a distribution function with a severe G-dwarf problem, which has very few metal-poor stars. Further, as mentioned above, merger/satellite disruption models are likely to generate a metal-weak tail to the thick disk, containing stars shredded from the satellite and accreted during the merger, in addition to any metal-poor stars in the original disk.

Note however that substantial pre-enrichment, which is the easiest way to reduce the expected number of metal-poor stars, appears unlikely. The low angular momentum of the stellar halo points to the central bulge as the recipient of gas ejected during the evolution of this component (Eggen, Lynden-Bell and Sandage 1962; Carney, Latham and Laird 1990; Wyse and Gilmore 1992) rather than the disk, and thus any such metalenriched gas is not available to 'pre-enrich' the thick disk. Predictions of the expected number of metal-weak thick disk stars are clearly very model-dependent, though some are expected in almost all models.

The metallicity distribution of the stellar halo has been defined by many samples, some kinematically selected, some not, with a concensus that it is adequately described mathematically by a Gaussian in log metallicity, of mean -1.5dex and of sigma 0.4 dex (e.g. Norris and Ryan 1989; Laird et al. 1988). The iron abundance distributions of the present paper indeed shows a local maximum at -1.5 dex. The kinematically-defined local thick disk has a metallicity distribution that can also be mathematically described by a Gaussian, of mean -0.6dex and of sigma 0.3dex (e.g. Carney et al. 1989). Several studies have identified stars with kinematics that assign them to the thick disk, but with $[\mathrm{Fe} / \mathrm{H}] \lesssim-1$ dex (e.g. Norris, Bessel and Pickles 1985; Morrison, Flynn and Freeman 1990; Morrison 1993a). What fraction of the metal-poor stars seen in the present sample can one ascribe to the thick disk and to the halo?

Even with no chemical abundance gradients, the expectation will depend on the distance of the sample under study, due to the different density profiles of the thick disk and of the halo. Locally, the relative normalisations of thick disk and halo to the thin disk are $\sim 0.04 / 0.00125$ or around a factor of 30 more thick disk stars than halo stars. To within the accuracy of the simple Gaussian model fits to the iron abundance distributions, $[\mathrm{Fe} / \mathrm{H}]=-1$ dex is equidistant in terms of sigma from the means of the thick disk and of the halo. THus combination with the local normalisation gives that at this metallicity the relative numbers of thick disk and halo stars locally should be around 30 , or that the vast majority of local stars with $[\mathrm{Fe} / \mathrm{H}] \sim-1$ dex should be associated with the thick disk rather than with the halo. For a typical scaleheight of $1 \mathrm{kpc}$ for the thick disk and an effective scaleheight of $4 \mathrm{kpc}$ for the halo, this ratio decreases to 10 at $1.5 \mathrm{kpc}$. 
Scaling from simple local normalisations and scaleheights to compare with the present number per unit volume at $1500 \mathrm{pc}$ is complicated due to the significantly different color-magnitude relations of the different stellar populations, which are most different in the color-apparent magnitude range selected. The direct predictions from star-count models for the actual observed sample are, as discussed in section 2 above, that the sample, as defined in color-apparent magnitude space, should contain $\sim 50 \%$ thick disk and $\sim 30 \%$ halo, or a ratio of 5:3 thick disk:halo.

The observed (but color-bias-corrected) SGP iron abundance distribution, as given in column 2 of Table 3, may be used to estimate the thick disk:halo ratio using the Gaussian fits, applied only over the range $\pm 1 \sigma$. Here the observed local maximum at $-0.6 \mathrm{dex}$ is rather broad, but taking the sum of three bins centered either on $-0.5 \mathrm{dex}$ or on -0.7 dex (which is $\pm 1 \sigma$ for the Gaussian fit to the local thick disk) gives $\sim 50$ stars. Summing three bins centered on $-1.5 \mathrm{dex}$, together with half of the next bins on either side, to give $\pm 1 \sigma$ for the halo, results in 10 stars. Thus assigning stars on the basis of an assumed Gaussian characterisation of the thick disk and halo metallicity distributions suggests a ratio of $\sim 5: 1$. This is a factor of $\sim 3$ fewer halo stars than predicted by the star-count models and a factor of $\sim 2$ more halo stars than is calculated from the simple Gaussian fitting argument above.

Admittedly the root- $\mathrm{N}$ uncertainty on this ratio is rather large, but the discrepancy between observations of halo stars and the star-count predictions is in broad agreement with that found by others (e.g. Friel 1987, 1988; Morrison 1993b). The breakdown of the star-count predictions could be much worse than this, allowing no quantitive statement to be made about the metal-poor stars in the thick disk. It is interesting however that there is increasing evidence both that the number of halo stars seen far from the plane is not consistent with expectations from commonly adopted halo number density normalisations and density profiles, and that the simple Gaussian description of abundance distribution functions is invalid. The kinematics of the stars will help provide a more definitive analysis, which is deferred to a future paper. 


\section{ACKNOWLEDGEMENTS}

The Center for Particle Astrophysics is supported by the NSF. RFGW acknowledges support from the AAS Small Research Grants Program in the form of a grant from NASA administered by the AAS, from the NSF (AST-8807799 and AST-9016226) and from the Seaver Foundation. Our collaboration was aided by grants from NATO Scientific Affairs Division and from the NSF (INT-9113306). GG thanks Mount Wilson and Las Campanas Observatories for access to their excellent facilities, as a Visiting Associate, during the early stages of this work.

\section{REFERENCES}

Aguilar, L., Carney, B., Latham, D. and Laird, J. 1994, AJ (in press)

Armandroff, T., Da Costa, G. S., Caldwell, N. and Seitzer, P. 1993, AJ, 106, 986

Bergbusch, P.A. and VandenBerg, D.A. 1992, ApJS, 81, 163

Bergh, S. van den 1962, AJ, 67, 486

Burstein, D. and Heiles, C. 1982, AJ, 87, 1165

Caldwell, N., Armandroff, T. E., Seitzer, P. and Da Costa, G. S. 1992, AJ, 103, 840

Carney, B. W., 1979, ApJ, 233, 211

Carney, B.W., Latham, D. and Laird, J. 1989, AJ, 97, 423

Carney, B.W., Latham, D. and Laird, J. 1990, AJ, 99, 572

Cayrel, R., Perrin, M.-N., Barbuy, B. and Buser, R. 1991a, A\&A, 247, 108

Cayrel, R., Perrin, M.-N., Barbuy, B., Buser, R. and Coupry, M.-F. 1991b, A\&A, 247, 122

Edmunds, M.G. 1990, MNRAS, 246, 678

Edvardsson, B., Andersen, J., Gustafsson, B., Lambert, D.L., Nissen, P. and Tomkin, J. 1993, A\&A, 275, 101

Eggen, O., Lynden-Bell, D. and Sandage, A. 1962, ApJ, 136, 748

Friel, E.D. 1987, AJ, 93, 1388

Friel, E.D. 1988, AJ, 95, 1727

Gilmore, G. 1984, in Astronomy with Schmidt-type Telescopes, edited by M. Capaccioli (Reidel, Dordrecht) p77

Reid, I.N. and Gilmore, G. 1982, MNRAS, 201, 73

Gilmore, G. and Reid, I.N. 1983, MNRAS, 202, 1025

Gilmore, G. Reid, I.N. and Hewett, P.C. 1985, MNRAS, 213, 257 
Gilmore, G. and Wyse, R.F.G. 1985, AJ, 90, 2015

Gilmore, G. and Wyse, R.F.G. 1986, Nature, 322, 806

Gilmore, G., Wyse, R.F.G. and Kuijken, K. 1989, ARA\&A, 27, 555

Gunn, J.E. 1987 in The Galaxy, edited by G. Gilmore and B. Carswell (Reidel, Dordrecht) p413

Hernquist, L and Quinn, P.J. 1993 in Galaxy Evolution: The Milky Way Perspective, edited by S. R. Majewski (ASP, San Francisco) p187

Iben, I. 1967, ARA\&A, 5, 571

Jones, J.B., 1991, Ph.D. thesis, Univ of Wales, Cardiff

Jones, J.B., Gilmore, G. and Wyse, R.F.G. 1994 (in preparation)

Kroupa, P., Tout, C.A. and Gilmore, G. 1991, MNRAS, 251, 293

Kroupa, P., Tout, C.A. and Gilmore, G. 1993, MNRAS, 262, 545

Kuijken, K. and Gilmore, G. 1989, MNRAS, 239, 605

Laird, J., Carney, B. and Latham, D. 1988 (LCL), AJ, 95, 1843

Laird, J., Rupen, M.P., Carney, B. and Latham, D. 1988, AJ, 95, 1908

Majewski, S.R. 1992, ApJS, 78, 87

Mihos, J.C. and Hernquist, L. 1994, ApJL, 425, L13

Morrison, H., 1993a, AJ, 105, 539

Morrison, H., 1993b, AJ, 106, 578

Morrison, H., Flynn C. and Freeman K.C., 1990, AJ, 100, 1191

Neese, C.L. and Yoss, K. 1988, AJ, 95, 463

Nissen, P.E. and Schuster, W. 1991, A\&A, 251, 457

Norris, J. and Ryan, S., 1989, ApJ, 340, 739

Norris, J. and Ryan, S., 1991, ApJ, 380, 403

Norris, J., Bessel, M. and Pickles, A. 1985, ApJS, 58, 463

Perrin, M.-N., Friel, E., Bienayme, O., Cayrel, R., Barbuy, B. and Boulon, J. 1994, $\mathrm{A} \& \mathrm{~A}($ in press)

Quinn, P.J., Hernquist, L. and Fullagher, D., 1993, ApJ, 403, 74

Rana, N.C. 1991, ARA\&A, 29, 129

Reid, I.N. and Gilmore, G. 1982, MNRAS, 201, 73

Ryan, S.G. and Norris, J.E. 1993, in Galaxy Evolution: The Milky Way Perspective, edited by S. R. Majewski (ASP, San Francisco) p103 
Schmidt, M. 1963, ApJ, 137, 758

Schuster, W.J. and Nissen, P.E. 1989, A\&A, 222, 69

Silk, J. and Wyse, R.F.G. 1993, Phy Rep, 231, 293

Stobie, R., Gilmore, G. and Reid, I.N. 1985, A\&AS, 60, 495

Stobie, R., Sagar, R. and Gilmore, G. 1985, A\&AS, 60, 503

Tinsley, B. 1980, Fund Cosmic Phys, 5, 287

Twarog, B.A. and Anthony-Twarog, B.J. 1994, AJ, 107, 1371

VandenBerg, D.A. 1985, ApJS, 58, 711

VandenBerg, D.A. and Bell, R.A. 1985, ApJS, 58, 561

White, S.D.M. 1980, MNRAS, 191, 1P

Wyse, R.F.G. and Gilmore, G. 1988, AJ, 95, 1404

Wyse, R.F.G. and Gilmore, G. 1992, AJ, 104, 144

Wyse, R.F.G. and Gilmore, G. 1994 (in preparation) 


\section{FIGURE CAPTIONS}

Figure 1(a) : B-V, V color-magnitude diagram for stars in the metallicity sample in the South Galactic Pole field.

Figure 1(b) : V-I, V color-magnitude diagram for stars in the metallicity sample in the South Galactic Pole field.

Figure $2: \mathrm{B}-\mathrm{V}, \mathrm{V}-\mathrm{I}$ two-color diagram for stars in the metallicity sample in the South Galactic Pole field, together with the ridge line for E-region standard stars from Reid and Gilmore (1982).

Figure 3 : B-V (circles) and V-I (squares) for one solar mass models, at fixed effective temperature, and age of many Gyr, from VandenBerg and Bell (1985; open symbols) and VandenBerg (1985; filled symbols) as a function of metallicity.

Figure $4: \mathrm{B}-\mathrm{V}, \mathrm{V}$ color-magnitude diagram for all stars in the chosen magnitude range in the SGP fields.

Figure 5(a) : B-V, V-I two-color diagram for stars in the metallicity sample in the South Galactic Pole field, with different ranges of metallicity plotted separately.

Figure 5(b) : B-V, V-I two-color diagram for the brighter stars $(\mathrm{V} \leq 17.30)$ in the metallicity sample in the South Galactic Pole field, with different ranges of metallicity plotted separately.

Figure 6 : Scatter plot of iron abundance versus $\mathrm{B}-\mathrm{V}$ color for the brighter stars in the SGP, together with the 12 Gyr turnoff points from VandenBerg and Bell (1985; crosses) and from VandenBerg (1985; asterisks).

Figure 7 : Comparison of the iron abundance histograms for the 'raw' bright SGP sample (dashed lines) and for the color-corrected and turnoff corrected bright sample (solid lines).

Figure 8: Scatter plot of iron abundance versus distance for the color-corrected and turnoff-corrected bright SGP sample.

Figure 9: Scatter plot of B-V versus distance for the SGP sample of Figure 8, together with the distance limits corresponding to the bright (dashed lines) and faint (solid lines) magnitude selection, for metallicities from left to right of -2 dex to solar, in steps of 0.5 dex.

Figure 10 : Histograms of the volume-complete iron abundance distributions for $\mathrm{F} / \mathrm{G}$ dwarfs in the SGP at 1500pc above the plane, derived by (i) assuming all stars follow the thick disk density profile, indicated by the solid lines, and (ii) assuming the most metal-poor bins are entirely stellar halo stars, and the most metal-rich are thin disk stars, as described in the text (dashed lines).

Figure 11(a) : B-V, V color-magnitude diagram for stars in the metallicity sample in F117. 
Figure 11(b) : V-I, V color-magnitude diagram for stars in the metallicity sample in F117.

Figure 12 : B-V, V color-magnitude diagram for stars in the relevant magnitude range in F117 fibre fields.

Figure 13 : B-V, V-I two-color diagram for stars in the metallicity sample in F117.

Figure 14 : B-V, V-I two-color diagram for stars in the metallicity sample in F117, with different ranges of metallicity plotted separately.

Figure 15 : Scatter plot of iron abundance versus B-V color for the stars in F117, together with the 12 Gyr turnoff points from VandenBerg and Bell (1985; crosses) and from VandenBerg (1985; asterisks).

Figure 16 : Scatter plot of iron abundance versus distance for the F117 stars.

Figure 17 : Scatter plot of B-V versus distance for the F117 stars, together with the distance limits corresponding to the bright (dashed lines) and faint (solid lines) magnitude selection, for metallicities from left to right of -2 dex to solar, in steps of $0.5 \mathrm{dex}$.

Figure 18 : Histograms of the volume-complete iron abundance distributions for $\mathrm{F} / \mathrm{G}$ dwarfs in F117 at 1000pc above the plane, derived by (i) assuming all stars follow the thick disk density profile, indicated by the solid lines, and (ii) assuming the most metal-poor bins are entirely stellar halo stars, and the most metal-rich are thin disk stars, as described in the text (dashed lines). Note that those bins with $[\mathrm{Fe} / \mathrm{H}] \lesssim-1$ dex should formally be treated as lower limits.

Figure 19 : Comparison of the F117 $z=1000$ pc iron-abundance distribution (solid histograms) and the SGP $z=1500$ pc distribution (dashed histograms). The left panel shows the derived distributions under the assumption that all stars follow the thick-disk density profile, while the right panel includes thin disk and stellar halo contributions. 
TABLE 1 : Relative error contributions in $[\mathrm{Fe} / \mathrm{H}]$ determination

\begin{tabular}{cccccc}
\hline \hline $\mathrm{T}_{\text {eff }}$ & Error in $\mathrm{T}_{\text {eff }} \Delta\left[\frac{\mathrm{Fe}}{\mathrm{H}}\right]_{\left[\frac{\mathrm{Fe}}{\mathrm{H}}\right]=-0.5} \Delta\left[\frac{\mathrm{Fe}}{\mathrm{H}}\right]_{\left[\frac{\mathrm{Fe}}{\mathrm{H}}\right]=-1.5} \Delta\left[\frac{\mathrm{Fe}}{\mathrm{H}}\right]_{\left[\frac{\mathrm{Fe}}{\mathrm{H}}\right]=-0.5} \Delta\left[\frac{\mathrm{Fe}}{\mathrm{H}}\right]_{\left[\frac{\mathrm{Fe}}{\mathrm{H}}\right]=-1.5}$ \\
& $\sigma_{\mathrm{V}-\mathrm{I}}=0.05$ & $\sigma_{\mathrm{V}-\mathrm{I}}=0.05$ & $\sigma_{\mathrm{V}-\mathrm{I}}=0.05$ & $\mathrm{~S} / \mathrm{N}=15 / \AA$ & $\mathrm{S} / \mathrm{N}=15 / \AA$ \\
\hline $5000 \mathrm{~K}$ & $140 \mathrm{~K}$ & 0.13 & 0.16 & 0.12 & 0.15 \\
$5500 \mathrm{~K}$ & $170 \mathrm{~K}$ & 0.13 & 0.16 & & 0.09 \\
$6000 \mathrm{~K}$ & $210 \mathrm{~K}$ & 0.14 & 0.17 & 0.25 \\
\hline \hline
\end{tabular}


TABLE 2: Observed and Derived Data for SGP stars

\begin{tabular}{|c|c|c|c|c|c|c|c|}
\hline ra (1950.0) & $\operatorname{dec}(1950.0)$ & $\mathrm{V}$ & $\mathrm{B}-\mathrm{V}$ & $\mathrm{V}-\mathrm{I}$ & {$[\mathrm{Fe} / \mathrm{H}]$} & $\operatorname{dist}(p c)$ & Teff \\
\hline 04739.40 & -291331.1 & 16.47 & 0.80 & 0.73 & 0.16 & 1478 & 5602 \\
\hline 04751.26 & -291014.5 & 16.67 & 0.62 & 0.93 & -0.29 & 2247 & 4961 \\
\hline $\begin{array}{lll}0 & 48 & 0.56\end{array}$ & $-2906 \quad 5.5$ & 17.24 & 0.62 & 0.84 & -0.78 & 2273 & 5231 \\
\hline $048 \quad 5.89$ & -285959.9 & 16.84 & 0.68 & 1.00 & -0.90 & 1553 & 4770 \\
\hline 04811.66 & $-2859 \quad 4.5$ & 15.90 & 0.50 & 0.63 & -0.84 & 1576 & 5989 \\
\hline 04817.96 & $-2941 \quad 2.1$ & 17.33 & 0.63 & 0.96 & -0.92 & 2165 & 4877 \\
\hline 04818.35 & $-29 \quad 0 \quad 56.9$ & 16.38 & 0.64 & 0.74 & -0.25 & 1907 & 5566 \\
\hline 04820.79 & -291652.8 & 16.97 & 0.64 & 0.80 & -0.30 & 2441 & 5360 \\
\hline 04825.88 & -292147.8 & 16.23 & 0.89 & 0.98 & -0.17 & 978 & 4823 \\
\hline 04826.71 & -294511.5 & 17.88 & 0.52 & 0.74 & -1.19 & 3164 & 5566 \\
\hline 04830.53 & -294844.9 & 16.29 & 0.49 & 0.65 & -0.72 & 2058 & 5908 \\
\hline 04834.00 & -295256.7 & 17.18 & 0.85 & 0.95 & 0.13 & 1800 & 4905 \\
\hline 04835.40 & -291414.5 & 17.31 & 0.63 & 0.88 & -0.56 & 2561 & 5107 \\
\hline 04836.67 & $-29 \quad 722.0$ & 15.97 & 0.70 & 0.77 & 0.03 & 1512 & 5461 \\
\hline 04841.27 & -294756.8 & 16.84 & 0.87 & 1.00 & -0.34 & 1270 & 4770 \\
\hline 04849.64 & -293616.9 & 17.92 & 0.46 & 0.75 & -0.67 & 4813 & 5531 \\
\hline 04854.26 & $-30 \quad 457.0$ & 17.25 & 0.78 & 1.02 & -0.75 & 1588 & 4718 \\
\hline $049 \quad 2.00$ & -293014.7 & 16.26 & 0.54 & 0.73 & -1.09 & 1501 & 5602 \\
\hline $049 \quad 3.07$ & -291526.9 & 16.29 & 0.74 & 0.81 & -0.50 & 1262 & 5327 \\
\hline $049 \quad 3.95$ & -293559.5 & 17.49 & 0.55 & 0.78 & -0.98 & 2723 & 5427 \\
\hline $049 \quad 6.70$ & -291833.4 & 16.35 & 0.64 & 0.74 & -0.22 & 1861 & 5461 \\
\hline $049 \quad 6.83$ & $-30 \quad 457.8$ & 16.48 & 0.86 & 0.90 & -0.29 & 1127 & 5048 \\
\hline 04912.50 & $-30 \quad 246.9$ & 15.79 & 0.65 & 0.68 & -0.60 & 1187 & 5789 \\
\hline
\end{tabular}


TABLE 2 (continued)

\begin{tabular}{|c|c|c|c|c|c|c|c|}
\hline ra (1950.0) & $\operatorname{dec}(1950.0)$ & $\mathrm{V}$ & $\mathrm{B}-\mathrm{V}$ & $\mathrm{V}-\mathrm{I}$ & {$[\mathrm{Fe} / \mathrm{H}]$} & $\operatorname{dist}(p c)$ & Teff \\
\hline 04914.60 & $-30 \quad 2 \quad 6.5$ & 16.82 & 0.76 & 0.92 & -0.87 & 1295 & 4990 \\
\hline 04918.01 & $-30 \quad 553.6$ & 16.30 & 0.71 & 0.79 & -0.11 & 1639 & 5393 \\
\hline 04918.32 & -293445.5 & 16.55 & 0.77 & 0.87 & -0.20 & 1519 & 5138 \\
\hline 04918.45 & $-30 \quad 947.0$ & 17.68 & 0.95 & 0.97 & -0.55 & 1405 & 4850 \\
\hline 04921.46 & -295410.5 & 16.87 & 0.63 & 0.86 & -0.35 & 2330 & 5168 \\
\hline 04923.26 & -295334.9 & 15.90 & 0.85 & 1.04 & -1.88 & 494 & 4667 \\
\hline 04924.78 & -295722.3 & 16.31 & 0.83 & 0.89 & 0.12 & 1270 & 5077 \\
\hline 04930.85 & -292451.7 & 16.78 & 0.70 & 0.80 & 0.21 & 2210 & 5360 \\
\hline 04941.81 & -292222.7 & 16.03 & 0.78 & 0.83 & -0.12 & 1205 & 5262 \\
\hline 04943.22 & -295448.9 & 17.27 & 0.57 & 0.84 & -0.96 & 2373 & 5231 \\
\hline 04947.37 & -295955.5 & 15.99 & 0.57 & 0.69 & -0.63 & 1554 & 5751 \\
\hline 04952.71 & -295819.5 & 16.08 & 0.69 & 0.79 & -0.56 & 1257 & 5393 \\
\hline $\begin{array}{lll}0 & 50 & 2.47\end{array}$ & -295235.9 & 16.86 & 0.63 & 0.78 & -0.66 & 1978 & 5427 \\
\hline $050 \quad 3.56$ & $-2957 \quad 2.5$ & 17.62 & 0.98 & 0.91 & -0.30 & 1404 & 5019 \\
\hline 05011.10 & -295758.0 & 17.69 & 0.30 & 0.70 & -1.80 & 3645 & 5713 \\
\hline $051 \quad 4.66$ & $-29 \quad 758.0$ & 16.84 & 0.58 & 0.89 & -0.38 & 2562 & 5077 \\
\hline 05114.84 & -285846.8 & 16.97 & 0.82 & 0.87 & -0.02 & 1735 & 5138 \\
\hline 05117.44 & $-29 \quad 827.0$ & 16.78 & 0.63 & 0.82 & -0.90 & 1696 & 5294 \\
\hline 05120.06 & $-29 \quad 630.2$ & 17.51 & 0.76 & 0.93 & -0.53 & 2079 & 4961 \\
\hline 05123.65 & $-29 \quad 455.1$ & 17.46 & 0.59 & 0.81 & -1.68 & 1852 & 5327 \\
\hline 05132.88 & -285644.7 & 16.90 & 0.67 & 0.82 & -0.73 & 1770 & 5294 \\
\hline 05140.45 & $-29 \quad 025.7$ & 16.95 & 0.56 & 0.71 & -0.78 & 2291 & 5676 \\
\hline 05141.08 & -291440.8 & 17.26 & 0.57 & 0.81 & -1.21 & 2108 & 5327 \\
\hline
\end{tabular}


TABLE 2 (continued)

\begin{tabular}{|c|c|c|c|c|c|c|c|}
\hline ra (1950.0) & $\operatorname{dec}(1950.0)$ & V & $\mathrm{B}-\mathrm{V}$ & $\mathrm{V}-\mathrm{I}$ & {$[\mathrm{Fe} / \mathrm{H}]$} & $\operatorname{dist}(p c)$ & Teff \\
\hline 05135.12 & -285316.6 & 17.60 & 0.34 & 1.01 & -1.28 & 3982 & 4744 \\
\hline 05152.94 & -285007.5 & 17.78 & 0.84 & 0.98 & -0.42 & 2036 & 4823 \\
\hline 05227.20 & $-29 \quad 639.0$ & 17.37 & 0.49 & 0.85 & -1.28 & 2570 & 5199 \\
\hline 05229.58 & -291721.5 & 16.95 & 0.86 & 0.91 & -0.41 & 1328 & 5138 \\
\hline 05235.17 & -285331.2 & 17.35 & 0.78 & 0.97 & -0.44 & 1918 & 5019 \\
\hline 05248.90 & -285928.6 & 17.58 & 0.89 & 0.99 & -1.13 & 1231 & 4850 \\
\hline 05257.14 & -291054.4 & 17.42 & 1.00 & 0.87 & -0.12 & 1301 & 4796 \\
\hline $053 \quad 6.62$ & -281748.7 & 17.30 & 0.73 & 0.96 & -0.36 & 2204 & 4877 \\
\hline 05337.02 & $-29 \quad 744.1$ & 17.01 & 0.96 & 0.76 & -0.21 & 1155 & 5496 \\
\hline 05347.39 & -282621.5 & 16.75 & 0.97 & 0.96 & -0.21 & 999 & 4877 \\
\hline $057 \quad 4.21$ & -295930.6 & 16.76 & 0.54 & 0.77 & -0.82 & 2155 & 5461 \\
\hline $057 \quad 8.76$ & $-30 \quad 530.7$ & 16.74 & 0.83 & 0.82 & 0.18 & 1548 & 5294 \\
\hline 05710.10 & $-2858 \quad 3.3$ & 17.10 & 0.54 & 0.68 & -0.69 & 2697 & 5789 \\
\hline 05714.53 & $-29 \quad 451.5$ & 17.05 & 0.56 & 0.74 & -0.49 & 2795 & 5566 \\
\hline 05729.50 & -285045.6 & 17.11 & 0.40 & 0.90 & -0.70 & 3759 & 5048 \\
\hline 05730.39 & -294917.0 & 16.66 & 0.61 & 0.77 & -0.94 & 1650 & 5461 \\
\hline 05730.54 & $-29 \quad 728.7$ & 16.90 & 0.58 & 0.74 & -0.31 & 2733 & 5566 \\
\hline 05732.88 & $-2755 \quad 0.5$ & 16.23 & 0.63 & 0.66 & -0.13 & 1931 & 5868 \\
\hline 05737.09 & $-29 \quad 750.0$ & 17.02 & 0.61 & 0.89 & -1.51 & 1536 & 5077 \\
\hline 05738.02 & $-30 \quad 843.6$ & 16.68 & 0.89 & 0.98 & -0.33 & 1127 & 4823 \\
\hline 05743.60 & $-28 \quad 344.3$ & 16.77 & 0.78 & 0.84 & -0.11 & 1701 & 5231 \\
\hline 05743.88 & $-29 \quad 433.0$ & 17.26 & 0.75 & 0.77 & -0.06 & 2345 & 5461 \\
\hline 05745.35 & $-30 \quad 92.1$ & 16.22 & 0.48 & 0.72 & -1.45 & 1441 & 5639 \\
\hline
\end{tabular}


TABLE 2 (continued)

\begin{tabular}{|c|c|c|c|c|c|c|c|}
\hline ra (1950.0) & dec (1950.0) & V & $\mathrm{B}-\mathrm{V}$ & $\mathrm{V}-\mathrm{I}$ & {$[\mathrm{Fe} / \mathrm{H}]$} & $\operatorname{dist}(p c)$ & Teff \\
\hline 05745.68 & -283716.3 & 16.26 & 0.84 & 0.85 & 0.09 & 1210 & 5199 \\
\hline 05745.72 & -295628.5 & 17.26 & 0.65 & 0.81 & -0.07 & 3022 & 5327 \\
\hline 05746.24 & -291015.2 & 17.66 & 0.75 & 0.80 & -0.10 & 2778 & 5360 \\
\hline 05748.92 & $\begin{array}{lll}-30 & 1 & 9.5\end{array}$ & 16.92 & 0.77 & 0.90 & -0.06 & 1904 & 5048 \\
\hline 05752.53 & -294438.6 & 16.78 & 0.52 & 0.83 & -0.76 & 2352 & 5262 \\
\hline 05755.16 & -295514.8 & 16.68 & 0.78 & 0.92 & -0.50 & 1370 & 4990 \\
\hline 05755.31 & -294323.2 & 16.02 & 0.63 & 0.81 & -0.88 & 1207 & 5327 \\
\hline 05757.45 & $-2848 \quad 45.2$ & 16.55 & 0.55 & 0.69 & -0.43 & 2350 & 5751 \\
\hline $058 \quad 0.10$ & -281555.0 & 17.22 & 0.66 & 0.80 & -0.95 & 1894 & 5360 \\
\hline $058 \quad 2.18$ & $-28 \quad 330.2$ & 17.25 & 0.74 & 0.80 & -0.21 & 2253 & 5360 \\
\hline $058 \quad 2.37$ & -295820.3 & 16.59 & 0.86 & 0.96 & -0.34 & 1160 & 4877 \\
\hline $058 \quad 3.16$ & -285532.6 & 17.03 & 0.51 & 0.63 & -0.54 & 3044 & 5989 \\
\hline $058 \quad 9.62$ & $-29 \quad 149.3$ & 17.42 & 0.50 & 0.68 & -0.67 & 3475 & 5789 \\
\hline 05810.50 & -284936.5 & 16.28 & 0.62 & 0.82 & -0.46 & 1720 & 5294 \\
\hline 05811.42 & -281450.7 & 16.28 & 0.54 & 0.72 & -0.60 & 1940 & 5639 \\
\hline 05813.39 & -28223.5 & 17.44 & 0.78 & 0.87 & -0.50 & 1944 & 5138 \\
\hline 05814.45 & $-29 \quad 847.7$ & 16.40 & 0.82 & 0.93 & -0.20 & 1249 & 4961 \\
\hline 05814.98 & -285928.4 & 17.22 & 0.45 & 0.61 & -1.24 & 2668 & 6073 \\
\hline 05819.20 & -295213.0 & 16.39 & 0.54 & 0.73 & -0.21 & 2517 & 5602 \\
\hline 05819.25 & $-2851 \quad 3.8$ & 16.76 & 0.53 & 0.67 & -0.45 & 2690 & 5828 \\
\hline 05819.46 & $-28 \quad 722.0$ & 16.38 & 0.68 & 0.81 & -0.43 & 1578 & 5327 \\
\hline 05819.58 & -275346.0 & 16.45 & 0.82 & 0.97 & -0.71 & 1017 & 4850 \\
\hline 05823.77 & $-28 \quad 1744.2$ & 16.96 & 0.77 & 0.82 & -0.31 & 1746 & 5294 \\
\hline
\end{tabular}


TABLE 2 (continued)

\begin{tabular}{|c|c|c|c|c|c|c|c|}
\hline ra $(1950.0)$ & $\operatorname{dec}(1950.0)$ & $\mathrm{V}$ & $\mathrm{B}-\mathrm{V}$ & $\mathrm{V}-\mathrm{I}$ & {$[\mathrm{Fe} / \mathrm{H}]$} & $\operatorname{dist}(p c)$ & Teff \\
\hline 05831.73 & -275535.7 & 16.82 & 0.82 & 0.86 & -1.56 & 877 & 5168 \\
\hline 05832.19 & -284834.8 & 17.62 & 0.50 & 0.83 & -0.94 & 3307 & 5262 \\
\hline 05837.01 & $-2825 \quad 0.0$ & 16.22 & 0.62 & 0.86 & -0.73 & 1457 & 5168 \\
\hline 05837.35 & -294127.8 & 16.12 & 0.68 & 0.79 & -0.38 & 1435 & 5393 \\
\hline 05839.88 & $-28 \quad 838.4$ & 17.90 & 0.57 & 0.82 & -1.27 & 2760 & 5294 \\
\hline 05840.18 & -295031.6 & 16.32 & 0.59 & 0.68 & -0.54 & 1807 & 5789 \\
\hline 05840.20 & $-30 \quad 056.8$ & 16.10 & 0.73 & 0.77 & -0.05 & 1452 & 5461 \\
\hline 05840.86 & -295934.7 & 17.70 & 0.67 & 0.82 & -1.05 & 2209 & 5294 \\
\hline 05841.22 & -274937.8 & 17.40 & 0.70 & 0.81 & -0.47 & 2355 & 5327 \\
\hline 05854.84 & $-28 \quad 217.9$ & 16.61 & 0.54 & 0.70 & -0.94 & 1894 & 5713 \\
\hline 05855.43 & -275125.3 & 17.34 & 0.80 & 0.86 & -0.18 & 2043 & 5168 \\
\hline 05857.74 & -295511.8 & 17.35 & 0.63 & 0.93 & -0.64 & 2504 & 4961 \\
\hline $\begin{array}{lll}0 & 59 & 2.18\end{array}$ & -281031.6 & 16.20 & 0.76 & 0.71 & 0.25 & 1448 & 5676 \\
\hline $059 \quad 5.41$ & -285713.5 & 16.39 & 0.86 & 0.98 & -0.21 & 1118 & 4823 \\
\hline $059 \quad 7.46$ & $-28 \quad 251.1$ & 17.44 & 0.58 & 0.97 & -1.26 & 2193 & 4850 \\
\hline $059 \quad 7.74$ & $\begin{array}{lll}-28 & 0 & 4.8\end{array}$ & 16.50 & 0.57 & 0.60 & -0.38 & 2246 & 6116 \\
\hline 05915.75 & -281238.5 & 17.24 & 0.62 & 0.90 & -1.09 & 1967 & 5048 \\
\hline 05924.51 & $-28 \quad 429.9$ & 17.11 & 0.53 & 0.66 & -0.68 & 2789 & 5868 \\
\hline 05925.38 & -275428.3 & 16.73 & 0.89 & 0.92 & -0.29 & 1173 & 4990 \\
\hline 05929.09 & -281317.7 & 16.55 & 0.64 & 0.63 & 0.00 & 2290 & 5989 \\
\hline 05936.01 & $-2741 \quad 1.9$ & 16.49 & 0.76 & 0.91 & -0.53 & 1300 & 5019 \\
\hline 05944.38 & -275355.0 & 16.91 & 0.85 & 0.87 & -0.36 & 1366 & 5138 \\
\hline 05953.23 & -273930.9 & 16.75 & 0.64 & 0.76 & -0.50 & 1992 & 5496 \\
\hline
\end{tabular}


TABLE 2 (continued)

\begin{tabular}{|c|c|c|c|c|c|c|c|}
\hline ra (1950.0) & $\operatorname{dec}(1950.0)$ & V & $\mathrm{B}-\mathrm{V}$ & $\mathrm{V}-\mathrm{I}$ & {$[\mathrm{Fe} / \mathrm{H}]$} & $\operatorname{dist}(p c)$ & Teff \\
\hline 05955.65 & -274439.0 & 16.63 & 0.50 & 0.71 & -0.46 & 2713 & 5676 \\
\hline 05956.84 & -274127.1 & 16.29 & 0.59 & 0.71 & -0.83 & 1536 & 5676 \\
\hline $\begin{array}{lll}1 & 0 & 1.83\end{array}$ & -274550.9 & 16.57 & 0.61 & 0.72 & -1.27 & 1369 & 5639 \\
\hline $\begin{array}{lll}1 & 0 & 3.58\end{array}$ & $-2731 \quad 4.6$ & 16.81 & 0.86 & 1.02 & -0.67 & 1111 & 4718 \\
\hline $\begin{array}{lll}1 & 0 & 3.72\end{array}$ & -275011.0 & 16.55 & 0.76 & 0.82 & -0.22 & 1544 & 5294 \\
\hline $\begin{array}{lll}1 & 0 & 12.17\end{array}$ & -273747.2 & 17.61 & 0.49 & 0.75 & -1.68 & 2454 & 5531 \\
\hline $1 \quad 032.51$ & -275356.7 & 16.64 & 0.71 & 0.91 & -1.13 & 1197 & 5019 \\
\hline $1 \quad 036.20$ & $-2742 \quad 0.1$ & 17.08 & 0.72 & 0.86 & -0.52 & 1889 & 5168 \\
\hline $1 \quad 042.13$ & $-2740 \quad 1.4$ & 17.48 & 0.80 & 0.82 & -0.40 & 1975 & 5294 \\
\hline $1 \quad 044.02$ & -275352.6 & 16.69 & 0.78 & 0.90 & -0.82 & 1190 & 5048 \\
\hline $1 \quad 044.44$ & $-2748 \quad 0.0$ & 17.48 & 0.82 & 0.82 & -0.49 & 1804 & 5294 \\
\hline 1046.68 & -274248.5 & 17.68 & 0.73 & 0.97 & -0.94 & 2001 & 4850 \\
\hline $\begin{array}{lll}1 & 0 & 48.19\end{array}$ & $-2745 \quad 16.8$ & 16.99 & 0.58 & 0.69 & -0.49 & 2589 & 5751 \\
\hline $\begin{array}{lll}1 & 052.27\end{array}$ & -275515.4 & 16.56 & 0.51 & 0.68 & -0.78 & 2153 & 5789 \\
\hline $1 \quad 055.22$ & $-2733 \quad 2.8$ & 16.35 & 0.68 & 0.64 & -0.40 & 1579 & 5948 \\
\hline $1 \quad 057.80$ & $-2723 \quad 5.7$ & 16.68 & 0.45 & 0.98 & -2.43 & 1421 & 4823 \\
\hline $\begin{array}{ll}1 & 1 \\
15.29\end{array}$ & -274626.5 & 16.59 & 0.87 & 0.90 & -0.16 & 1220 & 5048 \\
\hline $\begin{array}{lll}1 & 1 & 19.30\end{array}$ & -274419.3 & 17.38 & 0.62 & 0.75 & -0.54 & 2738 & 5531 \\
\hline
\end{tabular}


TABLE 3 : SGP Iron Abundance Distribution

\begin{tabular}{ccccccc}
\hline \hline Central $[\mathrm{Fe} / \mathrm{H}]$ & 1 & 2 & 3 & 4 & 5 & 6 \\
\hline+0.3 & 2 & 2 & 2290 & 1960 & 2 & 6 \\
+0.1 & 7 & 7 & 2290 & 1960 & 8 & 22 \\
-0.1 & 13 & 13 & 2180 & 2150 & 15 & 17 \\
-0.3 & 21 & 21 & 1995 & 1970 & 28 & 29 \\
-0.5 & 18 & 19 & 1810 & 1810 & 29 & 29 \\
-0.7 & 15 & 15 & 1640 & 1640 & 27 & 27 \\
-0.9 & 12 & 13 & 1500 & 1500 & 28 & 28 \\
-1.1 & 3 & 3 & 1370 & 1430 & 8 & 9 \\
-1.3 & 3 & 4 & 1260 & 1310 & 12 & 14 \\
-1.5 & 3 & 4 & 1170 & 1215 & 14 & 19 \\
-1.7 & 0 & 0 & 1095 & 1135 & 0 & 0 \\
-1.9 & 1 & 1 & 1035 & 1070 & 5 & 7 \\
-2.1 & 0 & 0 & 985 & 1015 & 0 & 0 \\
-2.3 & 0 & 945 & 970 & 0 & 12 \\
\hline \hline
\end{tabular}

Notes to TABLE 3

Column number 1 contains the 'raw' observed metallicity distribution for the brighter stars with $\mathrm{V}<17.30$ and $\mathrm{B}-\mathrm{V}<0.9$; column number 2 the $\mathrm{B}-\mathrm{V}$ bias-corrected and turnoff-corrected sample; column number 3 the characteristic distance (in pc) of each metallicity bin if all stars are assumed to follow the thick disk density profile; column number 5 the number of stars per unit volume at a fiducial distance of $1500 \mathrm{pc}$ under this assumed density law; column number 4 the characteristic distances if stellar halo and thin disk contributions are introduced, as explained in the text; column number 6 the number of stars per unit volume at 1500pc under these assumed density laws. 
TABLE 4 Observed and Derived Data for F117 stars

\begin{tabular}{|c|c|c|c|c|c|c|c|}
\hline ra (1950.0) & $\operatorname{dec}(1950.0)$ & $\mathrm{V}$ & $\mathrm{B}-\mathrm{V}$ & $\mathrm{V}-\mathrm{I}$ & {$[\mathrm{Fe} / \mathrm{H}]$} & $\operatorname{dist}(p c)$ & Teff \\
\hline 34005.00 & -591724.3 & 16.89 & 0.64 & 0.75 & -0.40 & 2236 & 5478 \\
\hline 34010.70 & -591732.3 & 17.27 & 0.63 & 0.71 & -0.77 & 2262 & 5620 \\
\hline 34024.33 & -593145.4 & 17.28 & 0.75 & 0.97 & -0.97 & 1569 & 4810 \\
\hline 34027.03 & -591611.7 & 17.78 & 0.62 & 0.95 & -2.34 & 1703 & 4864 \\
\hline 34029.41 & -600129.1 & 16.10 & 0.67 & 0.76 & -0.91 & 1125 & 5444 \\
\hline 34035.41 & -595517.5 & 16.25 & 0.59 & 0.65 & -0.85 & 1494 & 5848 \\
\hline 34036.46 & -591508.0 & 16.47 & 0.70 & 0.85 & -0.60 & 1440 & 5153 \\
\hline 34038.19 & -593054.7 & 16.14 & 0.66 & 0.64 & -1.33 & 982 & 5888 \\
\hline 34040.20 & -60 1009.4 & 16.65 & 0.77 & 0.85 & -0.75 & 1234 & 5153 \\
\hline 34105.77 & -592156.2 & 17.48 & 0.67 & 0.79 & -1.04 & 2005 & 5343 \\
\hline 34109.91 & -590932.8 & 16.31 & 0.90 & 0.89 & -0.19 & 982 & 5033 \\
\hline 34114.72 & -592648.5 & 16.27 & 0.72 & 0.69 & -0.23 & 1495 & 5694 \\
\hline 34114.10 & -600207.8 & 17.28 & 0.64 & 0.87 & -0.98 & 2011 & 5092 \\
\hline 34115.39 & -593454.9 & 16.26 & 0.87 & 0.91 & -0.11 & 1068 & 4975 \\
\hline 34121.52 & -600620.5 & 16.94 & 0.77 & 1.04 & -0.77 & 1397 & 4630 \\
\hline 34122.03 & -592433.7 & 17.01 & 0.75 & 0.80 & -0.62 & 1621 & 5311 \\
\hline 34130.33 & -600508.8 & 16.48 & 0.76 & 0.85 & -0.86 & 1112 & 5153 \\
\hline 34133.32 & -591332.3 & 16.30 & 0.59 & 0.75 & -1.91 & 1013 & 5478 \\
\hline 34133.79 & -591743.5 & 17.66 & 0.86 & 1.00 & -0.71 & 1615 & 4731 \\
\hline 34134.72 & -600426.7 & 17.48 & 0.63 & 0.80 & -0.82 & 2432 & 5311 \\
\hline 34136.49 & -594847.5 & 16.38 & 0.80 & 0.88 & -1.93 & 676 & 5063 \\
\hline 34141.81 & -600414.1 & 17.31 & 0.79 & 0.94 & -0.71 & 1623 & 4891 \\
\hline 34145.76 & -595408.6 & 16.48 & 0.73 & 0.79 & -0.63 & 1326 & 5343 \\
\hline
\end{tabular}


TABLE 4 (continued)

\begin{tabular}{|c|c|c|c|c|c|c|c|}
\hline ra (1950.0) & $\operatorname{dec}(1950.0)$ & $\mathrm{V}$ & $\mathrm{B}-\mathrm{V}$ & $\mathrm{V}-\mathrm{I}$ & {$[\mathrm{Fe} / \mathrm{H}]$} & $\operatorname{dist}(p c)$ & Teff \\
\hline 34149.99 & -601116.4 & 17.50 & 0.90 & 0.97 & -0.34 & 1597 & 4810 \\
\hline 34150.25 & -592229.8 & 16.73 & 0.73 & 0.86 & -0.63 & 1488 & 5122 \\
\hline 34152.43 & -594518.0 & 16.34 & 0.70 & 0.77 & -0.93 & 1162 & 5410 \\
\hline 34152.49 & -593519.7 & 16.12 & 0.66 & 0.73 & -0.33 & 1546 & 5549 \\
\hline 34159.53 & -594954.1 & 16.49 & 0.87 & 0.88 & -0.54 & 990 & 5063 \\
\hline 34202.62 & -600613.7 & 17.08 & 0.65 & 0.75 & -0.68 & 2067 & 5478 \\
\hline 34213.88 & -601233.1 & 17.38 & 0.57 & 0.85 & -0.81 & 2686 & 5153 \\
\hline 34214.96 & -591935.2 & 17.07 & 0.73 & 0.77 & -0.05 & 2270 & 5410 \\
\hline 34224.18 & -591234.9 & 16.57 & 0.64 & 0.76 & -0.84 & 1548 & 5444 \\
\hline 34237.74 & -600841.8 & 17.54 & 0.52 & 0.73 & -0.29 & 4310 & 5549 \\
\hline 34246.91 & -601048.8 & 17.40 & 0.81 & 0.90 & -0.55 & 1734 & 5004 \\
\hline 34247.76 & -594617.0 & 16.59 & 0.72 & 0.79 & -0.19 & 1764 & 5343 \\
\hline 34259.96 & -595913.4 & 17.87 & 0.61 & 0.90 & -1.01 & 2788 & 5004 \\
\hline 34302.04 & -592516.7 & 16.14 & 0.59 & 0.71 & -0.61 & 1604 & 5620 \\
\hline 34307.72 & -591244.3 & 17.20 & 0.64 & 0.70 & -0.42 & 2553 & 5657 \\
\hline 34307.28 & -60621.9 & 16.95 & 0.74 & 0.89 & -0.67 & 1578 & 5033 \\
\hline 34315.04 & -592020.2 & 17.46 & 0.79 & 0.76 & -0.68 & 1763 & 5444 \\
\hline 34316.47 & -594359.7 & 17.03 & 0.89 & 1.00 & -0.85 & 1063 & 4731 \\
\hline 34323.61 & -593427.1 & 17.75 & 0.64 & 0.87 & -2.43 & 1588 & 5092 \\
\hline 34328.02 & -593126.8 & 17.13 & 0.65 & 0.74 & -0.48 & 2339 & 5513 \\
\hline 34331.96 & -601546.0 & 16.09 & 0.67 & 0.74 & -0.57 & 1319 & 5513 \\
\hline 34333.59 & -590854.1 & 17.52 & 0.60 & 0.76 & -0.40 & 3300 & 5444 \\
\hline 34335.01 & -591038.8 & 17.58 & 0.81 & 0.97 & -0.55 & 1884 & 4810 \\
\hline
\end{tabular}


TABLE 4 (continued)

\begin{tabular}{|c|c|c|c|c|c|c|c|}
\hline ra (1950.0) & $\operatorname{dec}(1950.0)$ & $\mathrm{V}$ & $\mathrm{B}-\mathrm{V}$ & $\mathrm{V}-\mathrm{I}$ & {$[\mathrm{Fe} / \mathrm{H}]$} & $\operatorname{dist}(p c)$ & Teff \\
\hline 34337.73 & -595707.5 & 16.16 & 0.58 & 0.71 & -0.75 & 1542 & 5620 \\
\hline 34341.02 & -590641.0 & 16.18 & 0.57 & 0.68 & -0.89 & 1486 & 5732 \\
\hline 34341.58 & -600524.1 & 17.04 & 0.79 & 0.77 & -1.58 & 1029 & 5410 \\
\hline 34347.14 & -595131.0 & 16.43 & 0.76 & 0.81 & -0.56 & 1246 & 5278 \\
\hline 34350.94 & -592405.5 & 17.04 & 0.75 & 0.73 & -0.13 & 2063 & 5549 \\
\hline 34351.46 & -595001.5 & 17.23 & 0.64 & 0.80 & -0.29 & 2765 & 5311 \\
\hline 34356.92 & -591515.0 & 17.31 & 0.80 & 0.84 & -0.06 & 2110 & 5184 \\
\hline 34402.10 & -593013.6 & 16.27 & 0.68 & 0.73 & -0.59 & 1385 & 5549 \\
\hline 34404.90 & -592414.8 & 16.36 & 0.65 & 0.68 & -0.11 & 1965 & 5732 \\
\hline 34407.51 & -591452.6 & 16.77 & 0.86 & 0.85 & -0.27 & 1299 & 5153 \\
\hline 34411.51 & -591345.9 & 16.08 & 0.65 & 0.74 & -0.98 & 1131 & 5513 \\
\hline 34412.45 & -595249.2 & 16.94 & 0.63 & 0.79 & -0.54 & 2182 & 5343 \\
\hline 34413.68 & -590736.3 & 16.17 & 0.65 & 0.72 & -0.41 & 1558 & 5584 \\
\hline 34415.06 & -600607.3 & 17.28 & 0.77 & 0.80 & -0.06 & 2248 & 5311 \\
\hline 34423.46 & -595549.5 & 16.93 & 0.60 & 0.73 & -0.55 & 2324 & 5549 \\
\hline 34429.25 & -592307.0 & 16.43 & 0.81 & 0.78 & -0.49 & 1140 & 5376 \\
\hline 34745.83 & -60 1310.3 & 16.33 & 0.66 & 0.78 & -1.87 & 897 & 5376 \\
\hline 35006.66 & -601322.6 & 16.11 & 0.78 & 0.78 & -0.13 & 1245 & 5376 \\
\hline 35041.98 & -603236.2 & 16.93 & 0.69 & 0.91 & -1.35 & 1313 & 4975 \\
\hline 35046.34 & -603514.1 & 16.37 & 0.77 & 0.86 & -0.53 & 1200 & 5122 \\
\hline 35111.32 & -604426.3 & 16.37 & 0.69 & 0.80 & -0.49 & 1487 & 5311 \\
\hline 35133.91 & -604539.2 & 16.74 & 0.54 & 0.74 & -0.38 & 2702 & 5513 \\
\hline 35137.50 & -605226.4 & 16.22 & 0.84 & 0.86 & -0.58 & 924 & 5122 \\
\hline
\end{tabular}


TABLE 4 (continued)

\begin{tabular}{cccccccc}
\hline ra $(1950.0)$ & dec $(1950.0)$ & $\mathrm{V}$ & $\mathrm{B}-\mathrm{V}$ & $\mathrm{V}-\mathrm{I}$ & {$[\mathrm{Fe} / \mathrm{H}]$} & $\operatorname{dist}(\mathrm{pc})$ & $\mathrm{Teff}$ \\
\hline 35146.54 & -604129.1 & 16.18 & 0.78 & 0.70 & -0.48 & 1099 & 5657 \\
35150.00 & -602416.3 & 16.08 & 0.64 & 0.77 & -0.06 & 1808 & 5410 \\
35239.52 & -602108.3 & 16.23 & 0.65 & 0.78 & -0.05 & 1895 & 5376 \\
35303.53 & -604253.1 & 16.85 & 0.74 & 0.74 & -0.21 & 1874 & 5513 \\
35323.33 & -604631.7 & 16.58 & 0.55 & 0.69 & -1.19 & 1626 & 5694 \\
35342.11 & -603210.9 & 16.58 & 0.50 & 0.70 & -0.68 & 2347 & 5657 \\
35345.53 & -603808.6 & 16.52 & 0.85 & 0.85 & -0.14 & 1253 & 5153 \\
35353.94 & -605115.9 & 16.57 & 0.73 & 0.72 & -0.29 & 1628 & 5584 \\
35354.44 & -603528.5 & 16.90 & 0.74 & 0.80 & -0.36 & 1788 & 5311 \\
35440.75 & -604758.8 & 16.12 & 0.53 & 0.66 & -0.21 & 2279 & 5809 \\
35523.73 & -603514.1 & 16.07 & 0.67 & 0.72 & -0.26 & 1525 & 5584 \\
35939.11 & -601129.5 & 15.18 & 0.91 & 0.93 & 0.30 & 613 & 4919 \\
35959.63 & -601505.5 & 15.23 & 0.69 & 0.70 & 0.32 & 1111 & 5657 \\
35902.73 & -601449.7 & 15.06 & 0.60 & 0.67 & -0.69 & 914 & 5770 \\
35952.51 & -601810.7 & 15.90 & 0.77 & 0.87 & -0.61 & 931 & 5092 \\
35938.20 & -601919.6 & 15.38 & 0.57 & 0.70 & -0.67 & 1149 & 5657 \\
35901.68 & -602340.1 & 16.05 & 0.92 & 0.95 & -0.54 & 716 & 4864 \\
35813.33 & -601911.7 & 15.00 & 0.68 & 0.77 & 0.26 & 1026 & 5410 \\
\hline \hline
\end{tabular}


TABLE 5 : F117 Iron Abundance Distribution

\begin{tabular}{|c|c|c|c|c|c|}
\hline Central $[\mathrm{Fe} / \mathrm{H}]$ & 1 & 2 & 3 & 4 & 5 \\
\hline+0.3 & 2 & 2350 & 2035 & 2 & 6 \\
\hline+0.1 & 0 & 2350 & 2035 & 0 & 0 \\
\hline-0.1 & 11 & 2240 & 2210 & 12 & 13 \\
\hline-0.3 & 13 & 2045 & 2015 & 16 & 18 \\
\hline-0.5 & 17 & 1850 & 1850 & 26 & 26 \\
\hline-0.7 & 17 & 1670 & 1670 & 32 & 32 \\
\hline-0.9 & 12 & 1520 & 1520 & 28 & 28 \\
\hline-1.1 & 3 & 1390 & 1430 & 8 & 9 \\
\hline-1.3 & 2 & 1280 & 1315 & 7 & 7 \\
\hline-1.5 & 1 & 1190 & 1215 & 4 & 5 \\
\hline-1.7 & 0 & 1110 & 1135 & 0 & 0 \\
\hline-1.9 & 3 & 1045 & 1070 & 17 & 20 \\
\hline-2.1 & 0 & 990 & 1015 & 0 & 0 \\
\hline-2.3 & 1 & 950 & 970 & 7 & 9 \\
\hline-2.5 & 1 & 915 & 935 & 8 & 11 \\
\hline
\end{tabular}

Notes to TABLE 5

Column number 1 contains the 'raw' observed metallicity distribution for the stars with $0.5 \leq \mathrm{B}-\mathrm{V}<0.9$; column number 2 the characteristic distance (in pc) of each metallicity bin if all stars are assumed to follow the thick disk density profile; column number 4 the number of stars per unit volume at a fiducial vertical height above the plane distance of 1000pc under this assumed density law; column number 3 the characteristic distances if stellar halo and thin disk contributions are introduced, as explained in the text; column number 5 the number of stars per unit volume at $z=1000 \mathrm{pc}$ under these assumed density laws. 
TABLE 6 : Combined Iron Abundance Distribution at $Z=1500 \mathrm{pc}$

\begin{tabular}{crc}
\hline \hline Central $[\mathrm{Fe} / \mathrm{H}]$ & All Thick Disk & 3 Components \\
\hline+0.3 & 3 & 7 \\
+0.1 & 8 & 22 \\
-0.1 & 22 & 24 \\
-0.3 & 38 & 39 \\
-0.5 & 45 & 45 \\
-0.7 & 46 & 46 \\
-0.9 & 45 & 45 \\
-1.1 & 13 & 17 \\
-1.3 & 16 & 20 \\
-1.5 & 16 & 23 \\
-1.7 & 0 & 0 \\
-1.9 & 15 & 25 \\
-2.1 & 0 & 0 \\
-2.3 & 4 & 8 \\
-2.5 & 17 & 29 \\
\hline \hline
\end{tabular}

